\title{
Endogenous Nitric Oxide Is a Key Promoting Factor for Initiation of Seizure-Like Events in Hippocampal and Entorhinal Cortex Slices
}

\author{
Richard Kovács, ${ }^{1,3}$ Alexander Rabanus, ${ }^{1}$ Jakub Otáhal, ${ }^{1,4}$ Andreas Patzak, ${ }^{2}$ Julianna Kardos, ${ }^{3}$ Klaus Albus, ${ }^{1}$ \\ Uwe Heinemann, ${ }^{1}$ and Oliver Kann ${ }^{1}$ \\ Institutes for ${ }^{1}$ Neurophysiology and ${ }^{2}$ Vegetative Physiology, Charité-Universitätsmedizin Berlin, 10117 Berlin, Germany, ${ }^{3}$ Institute of Biomolecular \\ Chemistry, Chemical Research Center, Hungarian Academy of Sciences, 1025 Budapest, Hungary, and ${ }^{4}$ Institute of Physiology, Academy of Sciences of the \\ Czech Republic, 14220 Prague 4, Czech Republic
}

Nitric oxide (NO) modulates synaptic transmission, and its level is elevated during epileptic activity in animal models of epilepsy. However, the role of NO for development and maintenance of epileptic activity is controversial. We studied this aspect in rat organotypic hippocampal slice cultures and acute hippocampal- entorhinal cortex slices from wild-type and neuronal NO synthase (nNOS) knock-out mice combining electrophysiological and fluorescence imaging techniques. Slice cultures contained nNOS-positive neurons and an elaborated network of nNOS-positive fibers. Lowering of extracellular $\mathrm{Mg}^{2+}$ concentration led to development of epileptiform activity and increased NO formation as revealed by NO-selective probes, 4-amino-5-methylamino-2',7'-difluorofluorescein and 1,2diaminoanthraquinone sulfate. NO deprivation by NOS inhibitors and NO scavengers caused depression of both EPSCs and IPSCs and prevented initiation of seizure-like events (SLEs) in 75\% of slice cultures and 100\% of hippocampal-entorhinal cortex slices. This effect was independent of the guanylyl cyclase/cGMP pathway. Suppression of SLE initiation in acute slices from mice was achieved by both the broad-spectrum NOS inhibitor N-methyl-L-arginine acetate and the nNOS-selective inhibitor 7-nitroindazole, whereas inhibition of inducible NOS by aminoguanidine was ineffective, suggesting that nNOS activity was crucial for SLE initiation. Additional evidence was obtained from knock-out animals because SLEs developed in a significantly lower percentage of slices from $n N O S^{-1-}$ mice and showed different characteristics, such as prolongation of onset latency and higher variability of SLE intervals. We conclude that enhancement of synaptic transmission by NO under epileptic conditions represents a positive feedback mechanism for the initiation of seizure-like events.

\section{Introduction}

Since its first recognition as a signaling molecule in the CNS (Garthwaite et al., 1988; Bredt and Snyder, 1989), the free radical nitric oxide (NO) has been shown to be involved in synaptic transmission and plasticity (Prast and Philippu, 2001; Bon and Garthwaite, 2003), regulation of blood flow, mitochondrial respiration (Iadecola et al., 1995; Brown, 2001), and inflammatory processes (Good et al., 1996; Calabrese et al., 2000).

$\mathrm{NO}$ formation is also increased during epileptic seizures as a result of $\mathrm{Ca}^{2+}$-dependent activation of endothelial (eNOS) and neuronal (nNOS) NO synthases, as shown for kainic acid- and pentylene tetrazole-induced seizures in vivo (Mülsch et al., 1994;

Received Nov. 30, 2008; revised Jan. 14, 2009; accepted Feb. 2, 2009.

This work was supported by "Training and Excellence" Grant ICA1-CT-2002-70007, Hungarian Scientific Research Fund Grant F043589, Hungarian Ministry of Economics Grant MU00025/2002, and grants from the Deutsche Forschungsgemeinschaft (Sonderforschungsbereich 507 and TR3). We thank Dr. Sebastian Schuchmann for helpful discussion, Dr. Angela Skalweit for PCR analysis, and Jeannette Werner and Kristin Lehmann for excellent technical assistance.

Correspondence should be addressed to Dr. Oliver Kann, Neuronal Mitochondria Research Group, Institute for Neurophysiology, Charité-Universitätsmedizin Berlin, Tucholskystrasse 2, 10117 Berlin, Germany. E-mail: oliver.kann@charite.de.

DOI:10.1523/JNEUROSCI.5698-08.2009

Copyright $\odot 2009$ Society for Neuroscience $\quad$ 0270-6474/09/298565-13\$15.00/0
Kaneko et al., 2002; Gupta and Dettbarn, 2003; Kato et al., 2005), and in the low- $\mathrm{Mg}^{2+}$ model of epilepsy in vitro (Schuchmann et al., 2002). However, whether the increase of NO formation contributes to development and/or maintenance of epileptic activity is not yet fully understood. Contradictory results have been obtained in vivo, in which manipulation of the tissue NO level was either proepileptic or antiepileptic, depending on the experimental model of epilepsy (Kirkby et al., 1996; Wojtal et al., 2003). eNOS and likely nNOS are involved in seizure-associated changes in blood flow (de Vasconcelos et al., 2005), thereby increasing oxygen and glucose levels in areas with enhanced neuronal activity. Thus, any manipulation of the nitrergic system will also affect energy metabolism and consequently epileptic activity. To determine the direct effects of $\mathrm{NO}$ on seizure initiation, we chose an in vitro model of epilepsy, in which NO-dependent changes in tissue oxygenation were absent. Because previous controversial results might be ascribed to regional and interspecies differences in NO formation (Blackshaw et al., 2003), we compared the regulatory role of $\mathrm{NO}$ on epileptiform activity in different preparations from hippocampus and entorhinal cortex of rats and mice.

The close proximity of NMDA receptors and nNOS (Burette et al., 2002) couples neuronal activity and NO formation and 
allows localized transient NO signaling (Namiki et al., 2005; Sato et al., 2006) to its main targets, such as soluble guanylyl cyclase (sGC) (Szabadits et al., 2007) and mitochondria (Brown, 2001).

Here we combined the low- $\mathrm{Mg}^{2+}$ model of epilepsy and confocal fluorescence imaging of NO-sensitive probes, 4-amino-5methylamino-2', $7^{\prime}$-difluorofluorescein (DAF-fm) (Balcerczyk et al., 2005) and 1,2-diaminoanthraquinone sulfate (DAQ) (Schuchmann et al., 2002) to determine NO formation in hippocampal area CA3, which is a critical site for initiation of seizurelike events (SLEs) (Mody et al., 1987; Derchansky et al., 2006).

The effects of NO on epileptiform activity were tested by pharmacological manipulation of tissue NO level as well as by using a $n N_{O S}{ }^{-1-}$ mice (Huang et al., 1993). We provide evidence that nNOS-derived NO is a key promoting factor for the initiation of SLEs in the hippocampus and the entorhinal cortex, regardless of the animal species. Moreover, NO exerts its effect already before the first SLE.

\section{Materials and Methods}

Animals and slice preparation. Animal care and preparation were in accordance with the Helsinki declaration and institutional guidelines on transgenic animals, as approved by the local authority (T 0291/04, T 0003/06). Experiments were performed in rat organotypic hippocampal slice cultures and in acute hippocampal-entorhinal cortex slices from wild-type $(n=14)$ and $n N O S^{-1-}(n=9)$ mice. $129 \mathrm{~S}$ Nos $1^{\text {tmlPlh }}$ ( $\mathrm{NOS}^{-/-}$; Huang et al., 1993) and B6129F2/J wild-type mice were obtained from The Jackson Laboratory. The mice were bred as heterozygous $(+/-)$ mice by crossing $129 \mathrm{~S}$ Nos $1^{\mathrm{tm} 1 \mathrm{Plh}}$ and B6129F2/J wild-type mice. The breeding was continuously monitored by assessing the genetic status of the animals via PCR. The genetic testing using the tip of the mouse tail was done between postnatal days 14 and 20, according to protocols of The Jackson Laboratory (Patzak et al., 2008). Twenty- to 28-d-old mice were decapitated under deep ether anesthesia, and the brains were removed quickly. Transverse hippocampal-entorhinal cortex slices $(400 \mu \mathrm{m})$ were cut in ice-cold artificial CSF (ACSF) using a vibratome (Campden Instruments) and transferred to an incubation chamber. Slices were incubated in gassed $\left(95 \% \mathrm{O}_{2}, 5 \% \mathrm{CO}_{2}\right)$ ACSF for $2-4 \mathrm{~h}$ to recover from the preparation procedure before being used for experiments.

Slice cultures were prepared and maintained as described previously (Kovács et al., 2002). In brief, 7- to 8-d-old Wistar rat pups were decapitated, and the brains were removed and rinsed in ice-cold, gassed $(95 \%$ $\mathrm{O}_{2}, 5 \% \mathrm{CO}_{2}$ ) minimal essential medium (MEM). Slices $(400 \mu \mathrm{m})$ consisting of hippocampus and entorhinal cortex were cut (McIllwain Tissue Chopper; Mickle Laboratories) and placed on a culture plate insert (MilliCell-CM, $0.4 \mu \mathrm{m}$; Millipore) under sterile conditions. Slice cultures were used for experiments between 7 and $12 \mathrm{~d}$ in vitro. Half of the culture medium (containing 50\% MEM, 25\% HBSS, and 25\% horse serum, pH 7.4; all from Invitrogen) was replaced at every second day. Each experimental group was derived from at least three independent preparations.

Electrophysiology. Acute slices and slice cultures were transferred to the recording chambers mounted on epifluorescent microscopes (Olympus BX61WI; Olympus Europe) and superfused with ACSF $\left(5 \mathrm{ml} / \mathrm{min}, 33^{\circ} \mathrm{C}\right)$ (in mM): $129 \mathrm{NaCl}, 3 \mathrm{KCl}, 1.25 \mathrm{NaH}_{2} \mathrm{PO}_{4}, 1.8 \mathrm{MgSO}_{4}, 1.6 \mathrm{CaCl}_{2}, 21$ $\mathrm{NaHCO}_{3} 21$, and 10 glucose, $\mathrm{pH}$ 7.4. Epileptiform activity was induced by omitting of $\mathrm{Mg}^{2+}$ from ACSF (low- $\mathrm{Mg}^{2+}$ condition). Additionally, the extracellular $\mathrm{K}^{+}$concentration $\left(\left[\mathrm{K}^{+}\right]_{\mathrm{o}}\right)$ was elevated to $5 \mathrm{~mm}$ in slice cultures (Kovács et al., 2002).

Recording of local field potentials and changes in $\left[\mathrm{K}^{+}\right]_{\mathrm{o}}$ in the layers IV-V of the lateral entorhinal cortex were performed with doublebarreled $\mathrm{K}^{+}$-sensitive and reference microelectrodes, manufactured, and calibrated as described previously (Heinemann and Arens, 1992). In brief, electrodes were pulled from double-barreled theta glass (Science Products). The reference barrel was filled with $154 \mathrm{~mm} \mathrm{NaCl}$ solution, and the ion-sensitive barrel was filled with potassium ionophore I mixture A (60031; Fluka Chemie) and $100 \mathrm{~mm} \mathrm{KCl}$. Ion-sensitive microelec- trodes with a sensitivity of $59 \pm 2 \mathrm{mV}$ to a 10 -fold increase in $\left[\mathrm{K}^{+}\right]$were used for experiments. The home-built amplifier was equipped with negative capacitance feedback control that permitted dynamic recordings of $\left[\mathrm{K}^{+}\right]_{\mathrm{o}}$ with time constants of 50-200 ms. For testing slice viability, bipolar platinum wire electrodes were positioned medial to the recording electrode, and stimulus trains $(20 \mathrm{~Hz}, 10 \mathrm{~s})$ were applied before and after low- $\mathrm{Mg}^{2+}$ ACSF perfusion.

Local field potential and whole-cell patch-clamp recordings were performed in area CA3 of slice cultures by using a MultiClamp 700B amplifier (Axon CNS; Molecular Devices). Electrodes for local field potential recordings were filled with ACSF. Patch pipettes (4-5 M $\Omega$ ) were filled with a solution containing the following (in $\mathrm{mM}$ ): 135 potassium gluconate, $2 \mathrm{MgCl}_{2}, 0.1 \mathrm{CaCl}_{2}, 1$ EGTA, 10 HEPES, and $2 \mathrm{NaATP}, \mathrm{pH}$ 7.2, Visually identified pyramidal cells (input resistance, $166 \pm 7 \mathrm{M} \Omega$; $n=$ 43) were clamped to $-50 \mathrm{mV}$ after correction for the liquid junction potential. At this potential, glutamatergic synaptic currents were inwardly and GABAergic synaptic currents were outwardly directed, allowing for differentiation between EPSCs and IPSCs. Series resistance $(15.7 \pm 1 \mathrm{M} \Omega)$ was monitored online. Neurons showing $>25 \%$ change in series resistance were excluded from the analysis of synaptic events.

The following drugs were applied via the perfusion: aminoguanidine; 4-(carboxyphenyl)-4,4,5,5-tetramethylimidazole-1-oxyl 3-oxide (cPTIO); 4,4,5,5-tetramethylimidazole-1-oxyl 3-oxide (PTIO); $\mathrm{S}$-nitroso- $\mathrm{N}$-acetyl-DL-penicillamine (SNAP); $\mathrm{N}$-methyl-L-arginine acetate (L-NMMA); $N$-nitro-L-arginine methyl ester (L-NAME); $1 \mathrm{H}$-[1,2,4] oxadiazolo [4,3-a] quinoxalin-1-one (ODQ); and 7-nitroindazole (7$\mathrm{NI})$. PTIO, cPTIO, and ODQ were from Alexis Corporation. All other chemicals were from Sigma-Aldrich. If necessary, stock solutions were prepared in DMSO (final concentration of DMSO in ACSF, 0.05-0.1\%).

Fluorescence imaging. Fluorescence recordings were performed with confocal laser-scanning microscopes [Fluoview300 (Olympus Europe); Noran $\mathrm{Oz}$ (Prairie Technologies)] and photomultiplier-based microfluorimetry (Seefelder Messtechnik) and FeliX software (Photon Technology) by using $60 \times(0.9$ numerical aperture $)$ or $20 \times(0.5$ numerical aperture) water-immersion objectives. Slice cultures were stained in the incubator with the nonfluorescent, membrane-permeable diacetate ester form of the NO-indicator DAF-fm $(10 \mu \mathrm{M})$ in serum-free culture medium containing $0.6 \mathrm{~mm}$ L-arginine. Reaction with $\mathrm{NO}$ results in the fluorescent NO adduct of DAF-fm (DAF-fm fluorescence), which is membrane impermeable and accumulates in cells as revealed by images taken at different time points during staining $(20 \mathrm{~min}, 60 \mathrm{~min},>2 \mathrm{~h}$ ). Staining for $20 \mathrm{~min}$ was used in all experiments, which already allowed for differentiation between DAF-fm fluorescence and tissue autofluorescence (see below) while a large part of the dye was still in its NO-sensitive form. When monitoring DAF-fm fluorescence by confocal imaging (acquisition rate at $0.2 \mathrm{~Hz}$ for up to $90 \mathrm{~min}$ ), there was a linear decay of the fluorescence baseline because of photobleaching. Nevertheless, application of an external NO donor (SNAP, $100 \mu \mathrm{M}$ ) resulted in an immediate increase of DAF-fm fluorescence, indicating availability of a considerable fraction of NO-sensitive dye under these experimental conditions. It is noteworthy that DAF-fm fluorescence recovered to baseline after SNAP application. This recovery is not a true reversal, but it rather indicates increased susceptibility of the fluorescent $\mathrm{NO}$ adduct to photobleaching (Sheng et al., 2005) (see Fig. 2A). To minimize photo-oxidation and photobleaching of DAF-fm (Balcerczyk et al., 2005), we used a low acquisition rate $(0.2 \mathrm{~Hz})$ and minimized light exposure throughout the experiment. Changes in the fluorescence are presented as $\Delta f / f_{0}$ in percentage. Alterations in $\mathrm{NO}$ formation were determined by the steepness of the slope of DAF-fm fluorescence (control vs low- $\mathrm{Mg}^{2+}$ condition). The spatial pattern of NO formation under low- $\mathrm{Mg}^{2+}$ condition was determined by comparing the steepness of the slope in stratum radiatum versus stratum pyramidale after correction with the respective regional fluorescence baseline as determined under control condition in a subset of experiments. Oxidation reactions attributable to laser illumination did not affect the results because we determined fluorescence changes before and during epileptiform activity under identical illumination conditions. $\mathrm{NO}$ formation was not studied in NO-deprived slice cultures because cPTIO strongly quenched DAF-fm fluorescence.

Because the excitation and emission spectra of DAF-fm partially over- 
lap with that of flavin adenine dinucleotide (FAD), special care was taken to distinguish between both signals. Therefore, in a first series of experiments, fluorescence images were taken from unstained slice cultures $(n=4)$ at the optimum of FAD fluorescence (excitation at $450 \mathrm{~nm}$ ). Thereafter, excitation was changed to $488 \mathrm{~nm}$, and photomultiplier gain and offset were adjusted until FAD fluorescence disappeared. Subsequently, DAF-fm diacetate was applied $(10 \mu \mathrm{M})$ via the perfusion in normal ACSF. Under these conditions, any increase of the fluorescence corresponded to NO-dependent reaction of the dye. Indeed, DAF-fm fluorescence increased slowly and reached a plateau after $15 \mathrm{~min}$, indicating a basal NO formation in slice cultures. Unfortunately, such clear distinction between DAF-fm and FAD fluorescence was not possible in slice cultures that were stained in the incubator. Therefore, in a second set of experiments (see Fig. 3A), we compared changes in FAD fluorescence in unstained slice cultures $(n=5)$ with DAF-fm-stained slice cultures $(n=8)$ under control and low- $\mathrm{Mg}^{2+}$ conditions. SLEs were associated with transient biphasic changes of fluorescence in unstained and in DAF$\mathrm{fm}$-stained slice cultures, indicating that they originate from redox changes of FAD. In contrast, lasting changes in the slope steepness were only present in DAF-fm-stained slice cultures, thus clearly representing NO formation (see Fig. 3C). Additionally, we compared intracellular DAF-fm and FAD fluorescence pattern at the end of the experiments at high magnification. DAF-fm fluorescence pattern was homogenously distributed in the cytosol with highest accumulation in the nucleus, whereas FAD fluorescence pattern was dotted, indicating its mitochondrial origin (see Fig. 2C) (Huang et al., 2002). As an alternative to DAF$\mathrm{fm}$, we used the NO-sensitive dye DAQ (Schuchmann et al., 2002), which is nonfluorescent until having reacted with NO. The emission of the resulting $\mathrm{NO}$ adduct (excitation at $520 \mathrm{~nm}$; emission above $580 \mathrm{~nm}$ ) has no overlap with FAD autofluorescence. Slice cultures were stained with $15 \mu \mathrm{M}$ DAQ for $20 \mathrm{~min}$ in the incubator, and $1.5 \mu \mathrm{M}$ DAQ was continuously present in the perfusion during the whole experiment. After the photomultiplier-based microfluorimetry, slice cultures were transferred to the confocal microscope, and cellular distribution of the $\mathrm{NO}$ adduct of DAQ was investigated at higher magnification.

The reaction partner of DAF-fm and likely DAQ is not NO but rather peroxynitrite or $\mathrm{N}_{2} \mathrm{O}_{3}$ (Espey et al., 2001, Jourd'heuil, 2002). Hence, concomitant changes in superoxide formation might interfere with the DAF-fm (or DAQ) fluorescence. To exclude this possibility, we also monitored the kinetics of superoxide formation by using hydroethidine (HEt) (Kovács et al., 2002). Slice cultures were stained with $10 \mu \mathrm{M} \mathrm{HEt}$ for $20 \mathrm{~min}$ in the incubator, and fluorescence was excited at $530 \mathrm{~nm}$ and acquired above $580 \mathrm{~nm}$ under control and low- $\mathrm{Mg}^{2+}$ condition by using photomultiplier-based microfluorimetry.

Spectrophotometric determination of nitrite. For determination of nitrite in culture medium, slice cultures were exposed to normal or $\mathrm{Mg}^{2+}$ free $\mathrm{ACSF}$ in a $\mathrm{CO}_{2}$ incubator $\left(36.5^{\circ} \mathrm{C}\right)$. Slice cultures were washed twice with sterile ACSF and than transferred to a special six-well plate designed for electrophysiological recordings. To enhance nitrite accumulation, two slice cultures were grown on each culture plate insert. Tungsten microelectrodes were placed in the pyramidal cell layer of area CA3 under sterile conditions. Multiunit activity and alternating-currentcoupled field potentials were recorded in a $\mathrm{CO}_{2}$ incubator by using a home-built amplifier and Spike2 software (version 5.03; Cambridge Electronic Design) with a sampling rate of $11 \mathrm{kHz}$ (filter cutoff of $3 \mathrm{kHz}$ ). Half of the culture plate inserts on a six-well plate was exposed to low$\mathrm{Mg}^{2+}$ condition, whereas the other half served as control. Mechanical damage and infection rate of slice cultures was negligible, as revealed by long time $(24-48 \mathrm{~h})$ recordings of spontaneous activity (K. Albus, unpublished observation). Four hours after the onset of epileptiform activity, culture plate inserts were removed, ACSF was harvested, and nitrite content was determined by using the modified Griess diazotization reaction (Archer, 1993). Briefly, Griess reagent (100 $\mu$ l; Sigma-Aldrich), supernatant from slice cultures $(300 \mu \mathrm{l})$, and dionized water $(2.9 \mathrm{ml})$ were mixed and incubated for $30 \mathrm{~min}$. The absorbance was measured in a $1 \mathrm{ml}$ cuvette at $548 \mathrm{~nm}$ (UV spectrometer, DU 730; Beckman Coulter). For calibration, sodium nitrite solutions with concentrations between 0.1 and $100 \mu \mathrm{M}$ were prepared in deionized water. Absorbance measure- ments were performed using the standard nitrite solution in place of the experimental samples.

Immunostaining. NOS immunoreactivity was detected using a modified glucose oxidase-diaminobenzidine (DAB)-nickel method (Shu et al., 1988). In brief, slice cultures were fixed in $4 \%$ paraformaldehyde for $1 \mathrm{~h}$, followed by incubation $\left(3 \mathrm{~d}\right.$ at $\left.4^{\circ} \mathrm{C}\right)$ with diluted $(1: 1000)$ primary antibody (polyclonal anti-nNOS antibody, AB1552; Millipore Bioscience Research Reagents). After $1 \mathrm{~h}$ of incubation in biotinylated secondary antibody (1:100), slice cultures were washed in $\mathrm{AB}$ complex (Vector Laboratories) for $1 \mathrm{~h}$. For final oxidation of $\mathrm{DAB}$, a medium containing $\beta$-D-glucose, ammonium chloride, nickel ammonium sulfate, and glucose oxidase (Serva) was used. Slice cultures were counterstained by Vector Laboratories nuclear fast red dye.

Data evaluation. The distribution of nNOS-positive cells in slice cultures was analyzed with an upright microscope (AX-70; Olympus Europe) using the optical fractionator counting method, which is a combination of the fractionator sampling scheme and the disector counting technique (CastGrid System; Olympus Europe). The number of the nNOS-positive cells in hippocampal areas was determined in several counting frames $\left(1427.3 \mu \mathrm{m}^{2}\right)$ applied in systematically random manner ( $x$ and $y$ step $55 \mu \mathrm{m}$ ), focusing through the whole thickness of the tissue ( $100 \times$ oil-immersion objective, 1.35 numerical aperture). The final count was calculated using the optical fractionator form, and the final count of cells to unit area of a certain region was calculated using the point grid method (Gundersen, 1986). The entorhinal cortex was not included in the cell counting because it was preserved variably in slice cultures.

Spontaneous EPSCs and IPSCs were analyzed by using the event detection macro of the pClamp10 software (Axon CNS; Molecular Devices) in two data segments ( $5 \mathrm{~min}$, each), before and at the beginning of low$\mathrm{Mg}^{2+}$ condition. An average of 10-20 manually selected EPSCs (or IPSCs) served as a template, and the automatically detected events were verified by visual inspection. Clamping the cells at $-50 \mathrm{mV}$ allowed for simultaneous monitoring of EPSCs and IPSCs (Luhmann et al., 2000), without the use of pharmacological means that might have interfered with epileptiform activity or the effects of NO depletion. The frequency of the EPSCs at this potential was, however, somewhat underestimated as revealed by clamping the cells at the IPSC reversal potential, in which only EPSCs were visible. Nevertheless, monitoring EPSCs at $-50 \mathrm{mV}$ was still adequate for comparison of different treatments.

Multiple comparisons were done with one-way ANOVA and Tukey's post hoc tests. Paired Student's two-tailed $t$ test was used for comparison between two groups. SLE occurrence was compared by the Fisher's exact probability or Pearson's $\chi^{2}$ test (SPSS software package; SPSS Inc.). Statistical significance was defined as $p<0.05$. The data are presented as mean \pm SEM.

\section{Results}

\section{NOS activity in rat hippocampal slice cultures}

We first characterized the expression and distribution of nNOS in rat slice cultures by immunohistochemistry. The staining pattern revealed two types of nNOS-positive cells (Fig. 1). Type I displayed intense, homogeneous staining in both soma and dendrites (Fig. $1 A$ ). In contrast, type II showed a dotted staining exclusively in the soma, which limited more detailed morphological characterization (Fig. $1 \mathrm{~B}$ ). The stellate appearance of the majority of nNOS-positive cells corresponding to type I suggested that they were likely interneurons, which is in line with previous findings in the rat hippocampus (Valtschanoff et al., 1993; Blackshaw et al., 2003). However, a few pyramidal-shaped neurons were also present (Fig. 1C). An elaborated network of nNOSpositive fibers pervaded slice cultures, particularly in the subiculum (Fig. 1D). Type I and type II nNOS-positive cells were scattered in the principal cell layers of the hippocampus and the deep layers of the entorhinal cortex (Fig. 1E). The distribution of nNOS-positive cells in slice cultures was in agreement with the findings in vivo (Valtschanoff et al., 1993) (see also Keynes et al., 
2004). Cell counts revealed $33.2 \pm 3.9$ nNOS-positive cells in the dentate gyrus (DG), $36 \pm 7.4$ cells in area CA3, $15 \pm 3.36$ in area CA1 and $11 \pm 1.92$ in the subiculum (determined in $n=5$ slice cultures). Normalized density of nNOS-positive cells for each area is presented in Figure $1 F$. The density was highest in the DG and area CA3 $(p<0.01)$, suggesting that NO signaling might have a significant impact on neuronal activity in these areas, which are also critical for the development of SLEs.

We next determined NO formation in slice cultures by using fluorescent dyes (Brown et al., 1999; Schuchmann et al., 2002). Slice cultures were stained with DAF-fm, which is more sensitive to NO compared with its predecessor DAF-2, more photo-stable and less $\mathrm{pH}$ sensitive (Balcerczyk et al., 2005). The intracellular accumulation of membrane impermeable $\mathrm{NO}$ adduct of DAF-fm leads to a sustained fluorescence increase, which reflects NO formation (see Materials and Methods). Application of the NO donor SNAP (100 $\mu \mathrm{M})$ resulted in an additional increase in the fluorescence, indicating the presence of NO-sensitive, intracellular DAF-fm (Fig. 2A). Moreover, subsequent application of L-arginine $(2 \mathrm{mM})$ also increased DAF-fm fluorescence. This effect was blocked by previous application $(10 \mathrm{~min})$ of the broad-spectrum NOS inhibitor L-NMMA (200 $\mu \mathrm{M})$ (Fig. $2 A$ ). In addition, L-NMMA application alone led to a slight decrease in DAF-fm fluorescence $(-0.23 \pm 0.04 \% / \mathrm{min} ; n=2)$. Both findings indicate intrinsic NOS activity in slice cultures, which is in line with data obtained from acute hippocampal-entorhinal cortex slices (Schuchmann et al., 2002), and show that slice cultures represent a suitable model to study the effects of the nitrergic system on epileptiform activity.

We next investigated the spatial pattern of NO formation in area CA3 by applying confocal microscopy. In stratum pyramidale, DAF-fm accumulated in most of the pyramidal cell bodies (Fig. 2 B). In stratum radiatum, DAF-fm fluorescence originated predominantly from dendrites. Additionally, we also observed dye accumulation in smaller cell bodies, suggestive for astrocytes and microglial cells (Fig. 3C). Microglial cells were easily identified by morphology and membrane ruffling during the course of an experiment and showed a dotted fluorescence pattern (Fig. 2C, arrowheads), likely indicating phagocytosis of dye particles.

NO formation under low- $\mathrm{Mg}^{2+}$ condition in rat hippocampal slice cultures

The low- $\mathrm{Mg}^{2+}$ model of epilepsy is particularly suitable to study the effects of endogenous $\mathrm{NO}$ on epileptiform activity. In this model, removal of the extracellular $\mathrm{Mg}^{2+}$ ions reduces surface charge screening and facilitates activation of NMDA receptors,
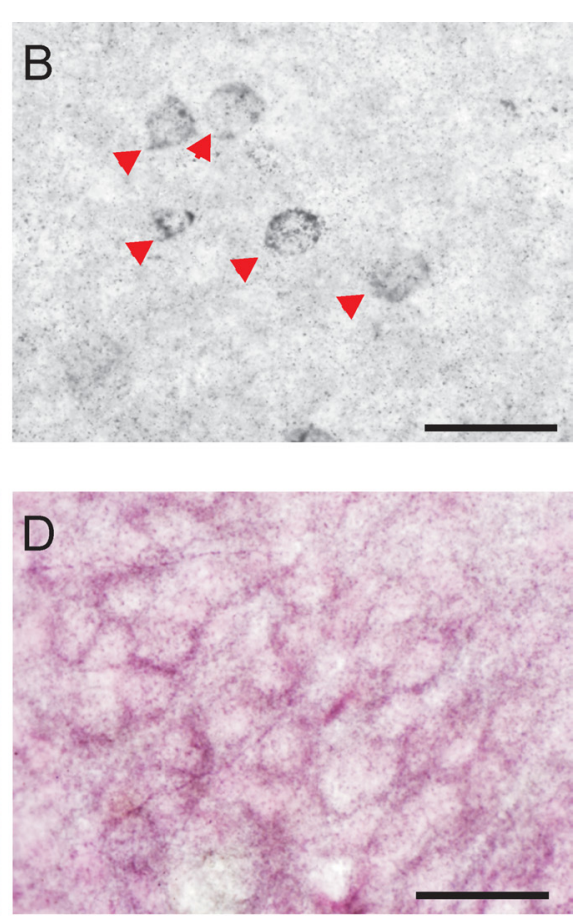

\section{F density of nNOS positive cells}

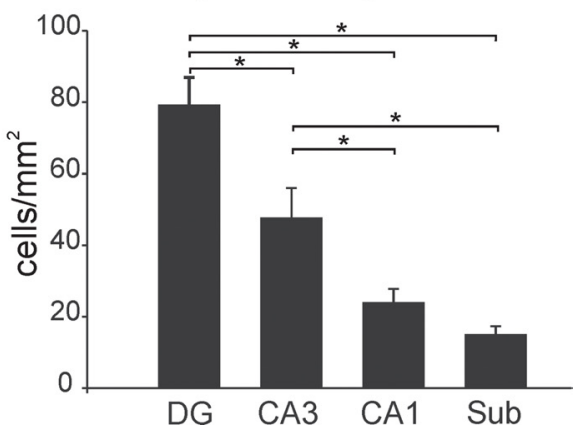

Figure 1. nNOS immunoreactivity in rat slice cultures. $\boldsymbol{A}$, Representative photomicrograph of type I nNOS-immunopositive neurons in area CA3 displaying intense somatic and dendritic staining. The majority of type I neurons had stellate appearance, somata but not in dendrites. C, Pyramidal-shaped neurons corresponding to type I (arrowheads) were only sparsely observed in the stratum pyramidale. $\boldsymbol{D}$, Dense network of nNOS-positive fibers was present throughout the whole slice culture, with the Distribution of nNOS-positive neurons (type I and II) in hippocampus and entorhinal cortex of a slice culture. $\boldsymbol{F}$, Summary diagram showing the density of nNOS-positive neurons in different areas of the hippocampus. Density of the nNOS-positive neurons was highest in DG and area CA3. Regional densities were calculated by using the point grid method in counts obtained from five slice cultures ( $95 \pm 7$ cells per slice culture; see Materials and Methods). Sub, Subiculum.

resulting in enhanced neuronal excitability and transmitter release as well as increased neuronal $\mathrm{Ca}^{2+}$ influx and activation of NOS (Mody et al., 1987; Garthwaite et al., 1988). Epileptiform activity developed in $100 \%$ of control rat slice cultures $(n=70)$ under low- $\mathrm{Mg}^{2+}$ condition. Epileptiform activity was classified in SLEs, interictal activity, and late recurrent discharges as described previously (Kovács et al., 2002). Additionally, we defined a "latent phase" during washout of $\mathrm{Mg}^{2+}$ ions before the first SLE. The latent phase was characterized by increased neuronal action potential firing, a slight positive direct current (DC) shift of the field potential as well as a robust increase in the frequency of synaptic currents in intracellular recordings (Fig. 3A) (see Fig. $4 B)$. The latent phase lasted for $11.57 \pm 1.2 \mathrm{~min}(n=70)$. The first SLE was followed by either an alternating sequence of recur- 
A

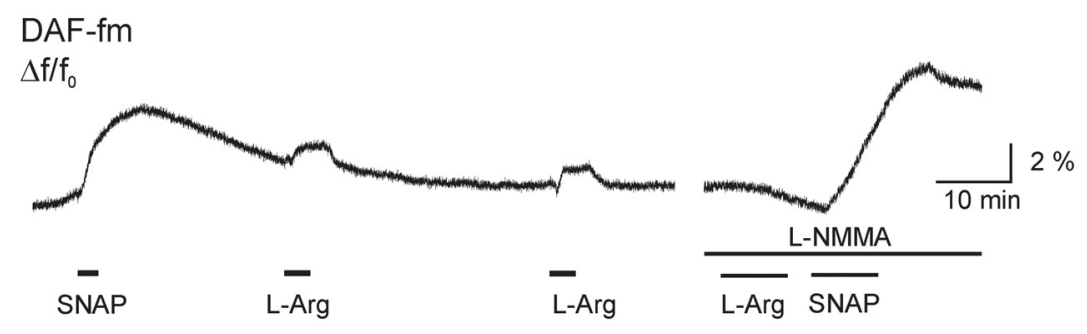

$\mathrm{B}$

DAF-fm, stratum pyramidale

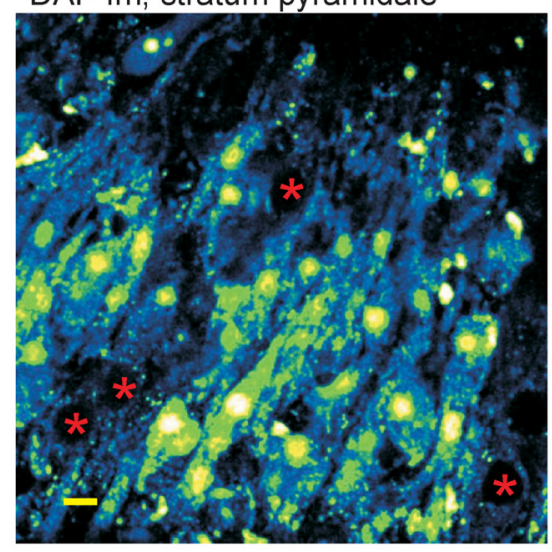

C

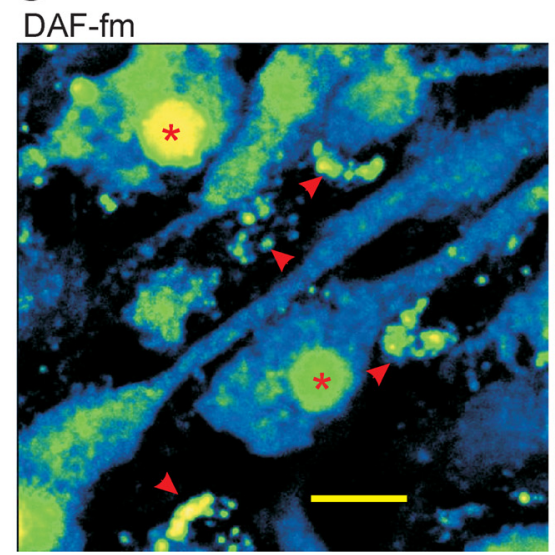

DAF-fm, stratum radiatum

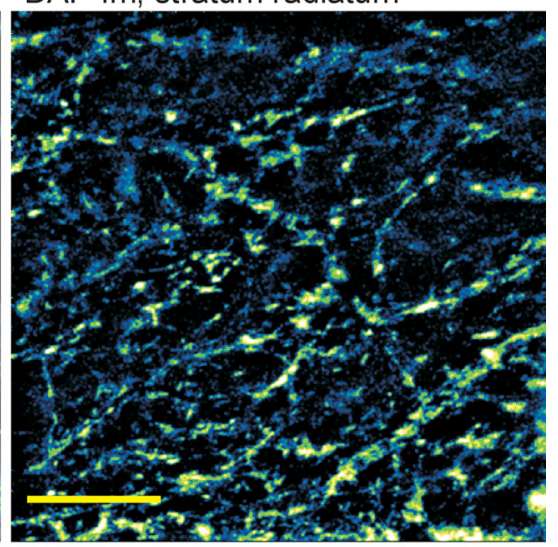

autofluorescence (FAD)

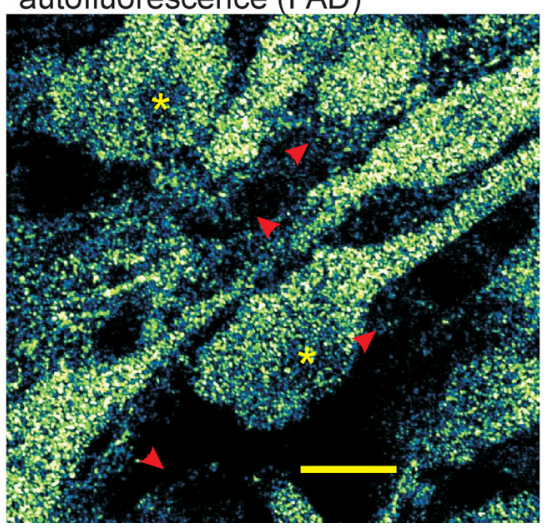

Figure 2. NO formation and DAF-fm staining pattern in rat slice cultures. $A$, Staining with the membrane-permeable diacetate form of DAF-fm revealed NO formation in slice cultures. Application of the exogenous NO donor SNAP (100 $\mu \mathrm{M})$ resulted in an increase in intracellular DAF-fm fluorescence. Subsequent applications of the NOS substrate L-arginine (2 mm; L-Arg) also caused a transient fluorescence increase (left trace), which was blocked after preincubation of the slice culture with the NOS inhibitor L-NMMA (200 $\mu$; r right trace). Application of SNAP in the presence of $\mathrm{L}-\mathrm{NMMA}$ still caused an increase in DAF-fm fluorescence. Fluorescence recordings were obtained with a $20 \times$ objective from the area $C A 3$ by using photomultiplier-based microflurimetry. $\boldsymbol{B}$, Representative confocal images from area CA3 of DAF-fm stained slice cultures. The fluorescent DAF-fm NO adduct accumulated in the cytosol and nucleus of most of the pyramidal cells in the stratum pyramidale (left). However, pyramidal cells with weak, dotted cytosolic staining were also observed (asterisks). In stratum radiatum, DAF-fm fluorescence appeared in dendrites and fibers (right). C, Comparison of DAF-fm (left) and FAD (right) autofluorescence in the same CA3 pyramidal cells. Images were obtained at the respective excitation maxima for DAF-fm ( $488 \mathrm{~nm}$ ) and FAD (450 $\mathrm{nm}$ ). Homogeneous DAF-fm fluorescence was observed in the cytosol and bright staining in the nucleus of pyramidal cells (asterisks). Additionally, small vesicles were present in the intracellular space (arrowheads), likely representing microglial phagocytosis of the dye. In contrast, autofluorescence originating from FAD showed a dotted staining pattern in the cytosol, which was weaker in the nucleus (asterisks) and absent in the small vesicles (arrowheads). The fluorescence was acquired as " $z$-stack," and three-dimensional projection was reconstructed from 5 to 10 focal planes $(0.5-1 \mu \mathrm{m}$ steps). Scale bars, $10 \mu \mathrm{m}$.

rent SLEs and interictal activity or late recurrent discharges (data not shown).

NO formation was detected by confocal imaging of DAF-fm fluorescence under control and low- $\mathrm{Mg}^{2+}$ conditions. In stra- tum radiatum, the slope of the DAF-fm fluorescence was negative in normal ACSF and increased significantly in low- $\mathrm{Mg}^{2+}$ $\operatorname{ACSF}(-1.3 \pm 0.3$ vs $-0.3 \pm 0.2 \% / \mathrm{min}$; $n=8$ slice cultures; $p=0.017)$, indicating an increase in $\mathrm{NO}$ formation relative to photobleaching (Fig. 3A). In contrast, in stratum pyramidale, there was no significant difference in the slope of the DAF-fm fluorescence under both conditions $(-0.54 \pm 0.1$ vs $-0.54 \pm 0.13 \% / \mathrm{min} ; n=$ $8 ; p=1)$. This finding was confirmed by comparing stratum radiatum and stratum pyramidale after regional baseline correction in a second set of experiments $(0.93 \pm$ 0.5 vs $0.06 \pm 0.3 \% / \mathrm{min} ; n=20$ slice cultures; $p<0.001$ ) (Fig. $3 C, D$ ). These data show that the rate of $\mathrm{NO}$ formation under low- $\mathrm{Mg}^{2+}$ condition increased particularly in the synaptic compartment.

In stratum radiatum, the increase in DAF-fm fluorescence started already during the latent phase, suggesting that facilitation of NMDA receptor activation under low- $\mathrm{Mg}^{2+}$ condition was sufficient to enhance NO formation (Fig. 3A). Additionally, SLEs were associated with fast, biphasic fluorescence transients that represented a crosstalk with FAD autofluorescence rather than fast NO formation because such transients were also observed in unstained slice cultures $(n=5)$ (Fig. $3 B$ ) (see Materials and Methods). In contrast to DAF-fm fluorescence, there was no change in the slope of the FAD fluorescence when switching from normal to low$\mathrm{Mg}^{2+}$ ACSF. Moreover, the fluorescence patterns of DAF-fm and mitochondrial FAD were clearly different in neurons (Fig. 2C) (Huang et al., 2002).

To exclude that FAD contributed to the DAF-fm signal, we also used the red fluorescent NO-sensitive probe DAQ (Schuchmann et al., 2002). DAQ fluorescence increased continuously both in normal ACSF and under low- $\mathrm{Mg}^{2+}$ condition (Fig. 3E). Similarly to DAF-fm, there was a significant increase in the slope of the DAQ fluorescence under low- $\mathrm{Mg}^{2+}$ condition starting already during the latent phase $(0.66 \pm 0.08$ vs $0.82 \pm 0.06 \% / \mathrm{min}$ in normal ACSF and under low- $\mathrm{Mg}^{2+}$ condition, respectively; $n=12$ slice cultures; $p=0.026$ ), indicating increased NO formation. This effect was reversible because perfusion with normal ACSF resulted in a significant decrease in the slope of DAQ fluorescence $(0.22 \pm 0.07 \%$ / $\min ; p<0.001)$. The NO adduct of DAQ accumulated homogeneously in the soma as well as in the dendrites of CA3 cells (Fig. 3F). In contrast to DAF-fm, DAQ fluorescence was completely absent in the nucleus, indicating that nuclear accumulation of DAF-fm presumably represents redistribution of the benzotriazole derivative (Nagano, 1999). 
A
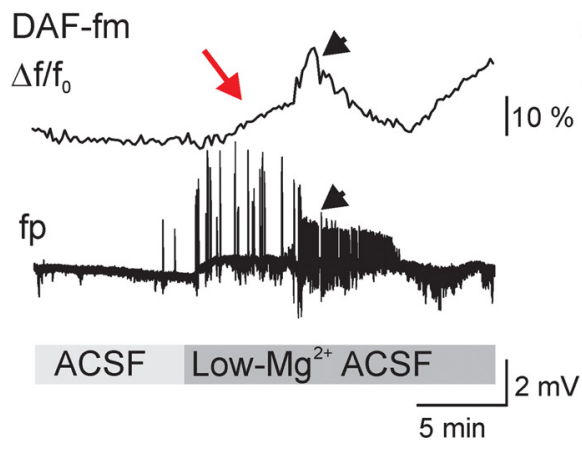

autofluorescence (FAD)

$\Delta \mathrm{f} / \mathrm{f}_{0}$
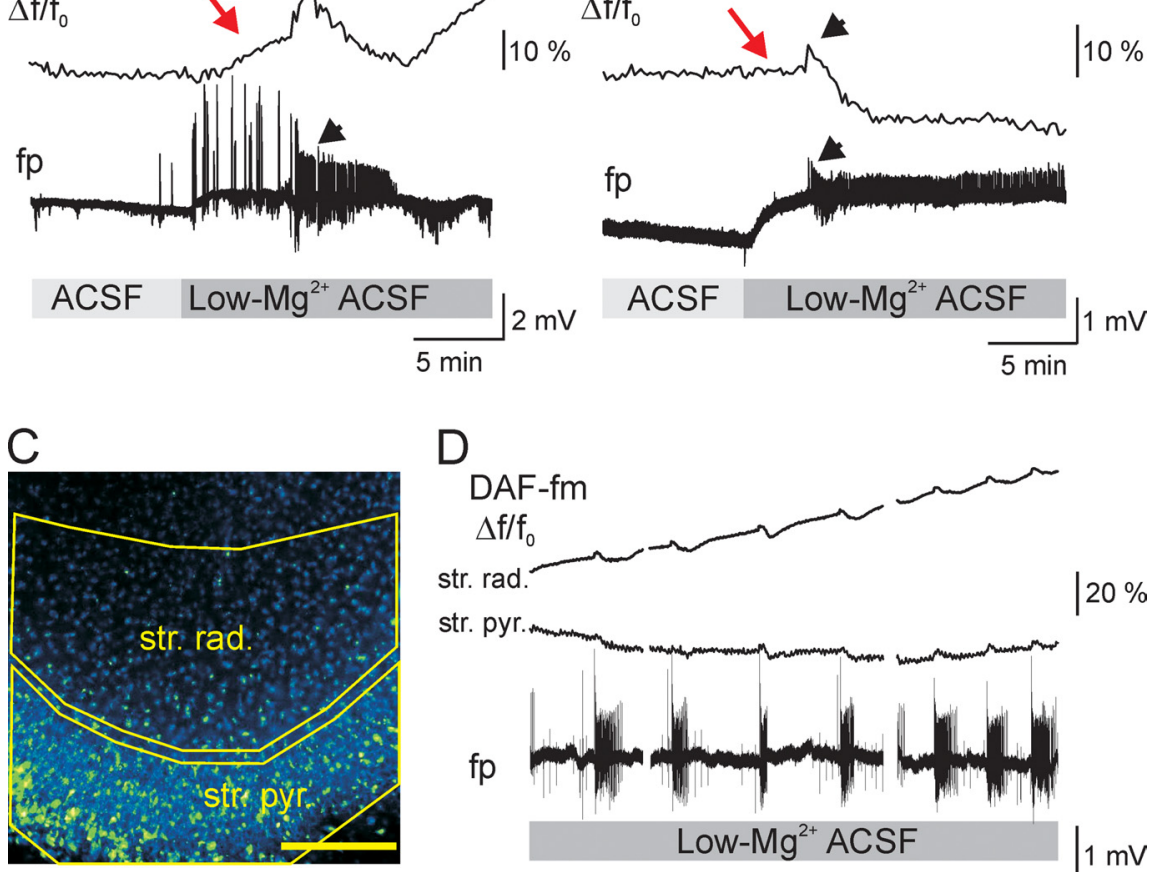

$\mathrm{D}$

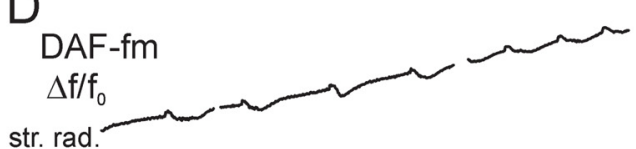

str. pyra

$\mathrm{fp}$

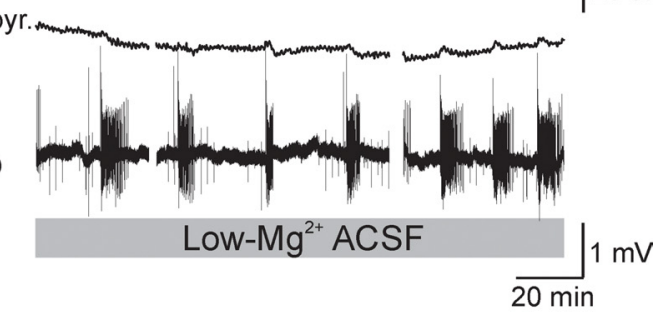

$\mathrm{E}$

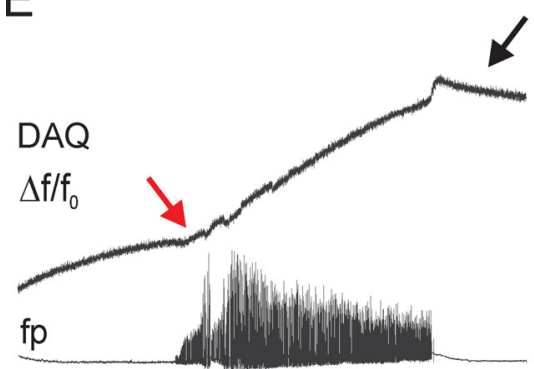

$\mathrm{F}$

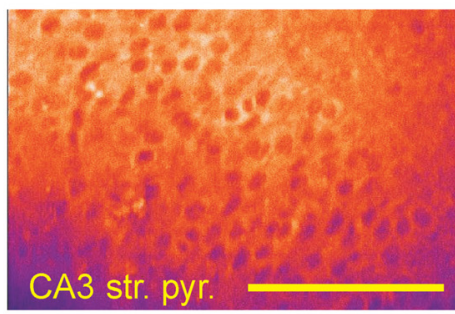

ACSF LOW-Mg ${ }^{2+}$ ACSF ACSF $2 \frac{2 m V}{30 \min }$
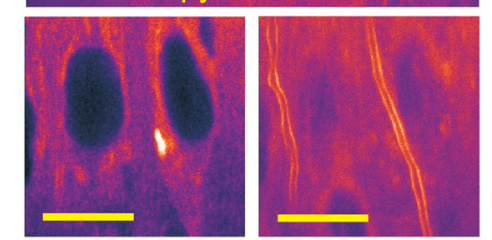

G

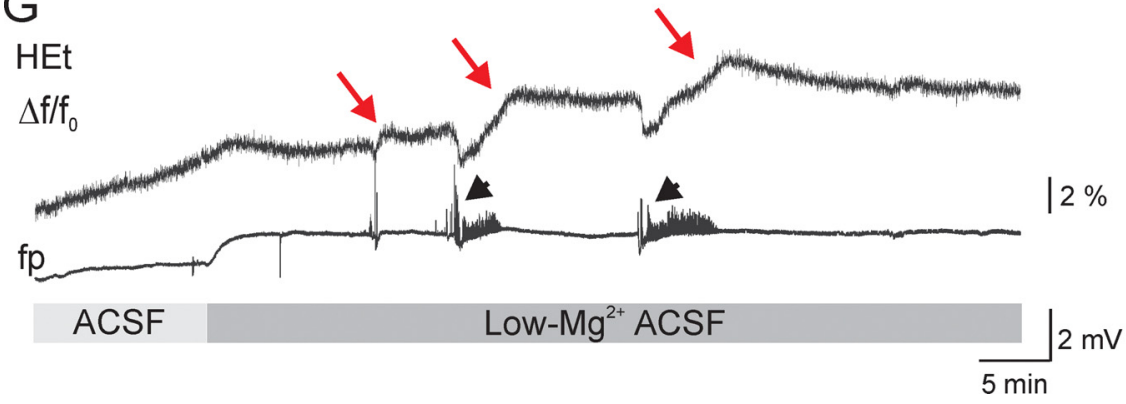

Figure 3. NO formation under low- $\mathrm{Mg}^{2+}$ condition in rat slice cultures. $A$, Changes in DAF-fm fluorescence as measured in the stratum radiatum during transition from control to low- $\mathrm{Mg}^{2+}$ condition. The slope of the DAF-fm fluorescence (red arrow, top trace on the left) became steeper under low- $\mathrm{Mg}^{2+}$ condition before the first SLE (local field potential, bottom trace), followed by a biphasic fluorescent transient during the SLE (arrowheads). $\boldsymbol{B}$, Changes in FAD fluorescence as measured in the stratum radiatum during transition from control to low-Mg ${ }^{2+}$ condition. In contrast to the DAF-fm fluorescence, the slope of FAD autofluorescence (red arrow, top trace on the right) remained stable during the latent phase before the first SLE. However, SLE-associated biphasic fluorescence transients were still present (arrowheads). C, Confocal image of area CA3 of a DAF-fm-stained slice culture, depicting the regions of interest (stratum radiatum and stratum pyramidale) in which NO formation was monitored. Scale bar, $200 \mu \mathrm{m}$. D, Regional differences in the DAF-fm fluorescence slope (top traces) during epileptiform activity (bottom trace). DAF-fm fluorescence increased in the stratum radiatum but not in the stratum pyramidale, indicating NO formation in the synaptic compartment.
The reaction of both DAF-fm and DAQ with NO might be influenced by the presence of superoxide and subsequent formation of peroxynitrite (Jourd'heuil, 2002). Therefore, we also measured superoxide formation by monitoring changes in HEt fluorescence. In contrast to DAF-fm and DAQ, the slope of HEt fluorescence decreased significantly during the latent phase $(0.37 \pm 0.14$ vs $-0.05 \pm 0.02 \% / \mathrm{min}$ in normal ACSF and under low- $\mathrm{Mg}^{2+}$ condition, respectively; $n=7$ slice cultures; $p<0.001$ ) (Fig. $3 G$ ), thus excluding the possibility that increased superoxide formation contributed to the rise in DAF-fm or DAQ fluorescence. Moreover, significant increases in superoxide formation occurred only transiently after SLEs $(-0.05 \pm 0.02$ vs $0.87 \pm 0.3 \% / \mathrm{min}$ during the latent phase and after SLEs, respectively; $p=0.001)$, in line with our previous data (Kovács et al., 2002). Thus, even if we consider peroxynitrite or other reactive nitrogen species as the reaction partners for DAF-fm and DAQ, the increase in their fluorescence is related to increases in $\mathrm{NO}$ rather than in superoxide formation.

The major pathway for NO metabolism in physiological solutions is the stepwise oxidation to nitrite and nitrate. Nitrite remains stable in solution for several hours, allowing detection by using the modified Griess reaction (Archer, 1993). Because perfusion with ACSF in the recording chamber would dilute nitrite below the detection limit of the method, we developed a new technique to induce and monitor epileptiform activity in slice cultures kept in six-well plates in the incubator under

$\leftarrow$

The slope of DAF-fm fluorescence was corrected for photobleaching by using the average regional fluorescence baseline as obtained under control condition ( $n=8$ slice cultures). $\boldsymbol{E}$, Changes in DAQ fluorescence as measured in the stratum radiatum during transition from control to low- $\mathrm{Mg}^{2+}$ condition. The slope of the DAQ fluorescence (red arrow, top trace) became steeper already during the latent phase (local field potential, bottom trace), indicating enhanced NO formation under low- $\mathrm{Mg}^{2+}$ condition. Wash-in of normal ACSF resulted in a decrease in the slope of $D A Q$ fluorescence (black arrow). $\boldsymbol{F}$, Confocal images of area CA3 in a DAQ-stained slice culture showing homogeneous accumulation of the fluorescent NO adduct of DAQ (top). Cytosol and dendrites but not the nuclei were strongly stained with DAQ (bottom). Scale bars: top, 200 $\mu \mathrm{m}$; bottom, $10 \mu \mathrm{m}$. G, Changes in HEt fluorescence as measured in the stratum radiatum during transition from control to low- $\mathrm{Mg}^{2+}$ condition. The slope of HEt fluorescence (top trace) decreased under low-Mg ${ }^{2+}$ condition before the first SLE (local field potential, bottom trace). SLEs were associated by biphasic changes in HEt fluorescence (red arrows), indicating transient superoxide formation selectively after SLEs (arrowheads). fp, Field potential; str. rad., stratum radiatum; str. pyr., stratum pyrimidale. 


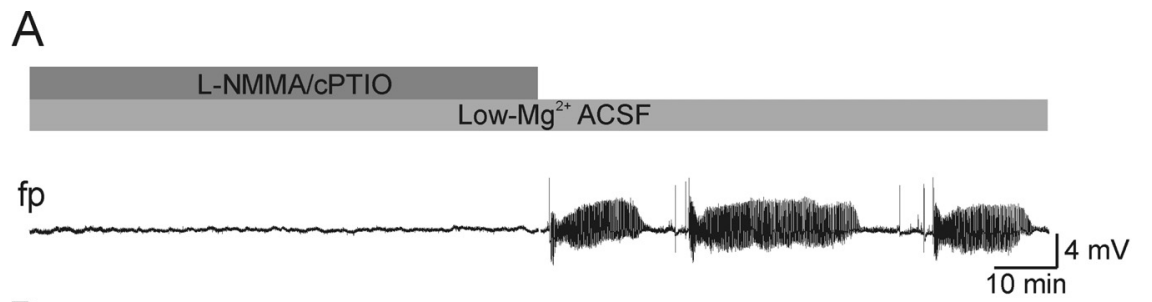

$\mathrm{B}$

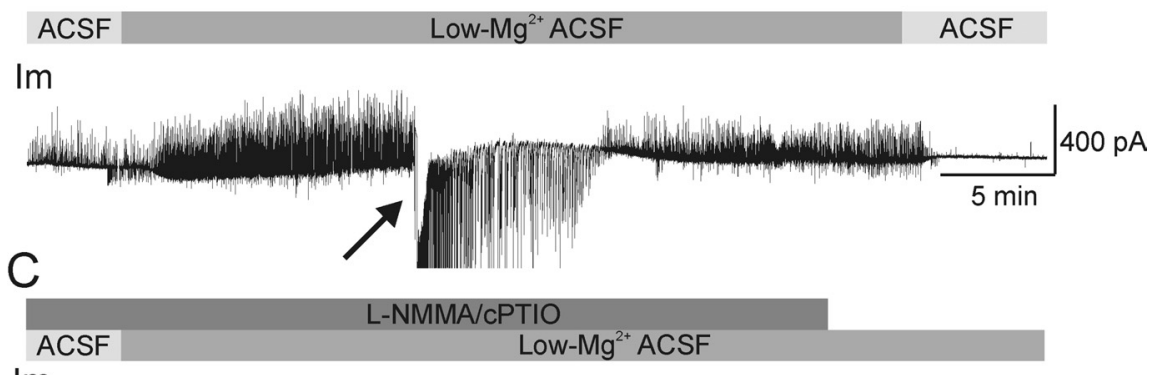

Im

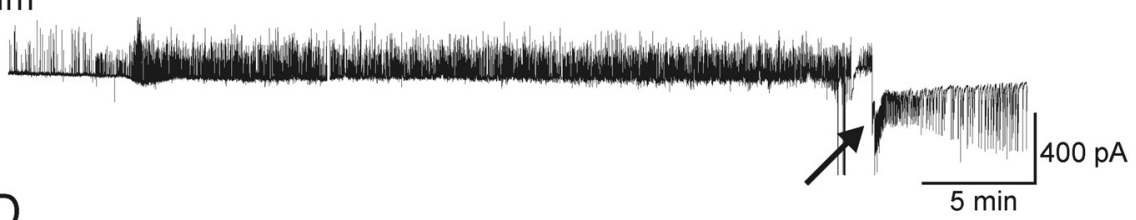

Interictal activity in the presence of L-NMMA/cPTIO Im

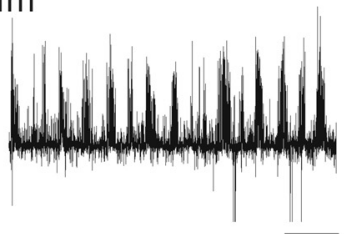

$\overline{20 \mathrm{~s}}$

$\mathrm{E}$

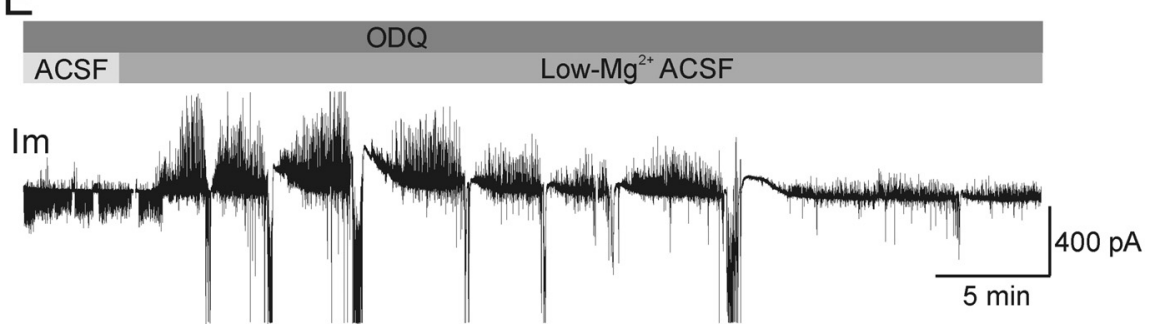

Figure 4. Effects of NO deprivation and $\mathrm{SGC}$ inhibition on the epileptiform activity in rat slice cultures. $A$, Deprivation of NO by coapplication of the NOS inhibitor L-NMMA $(200 \mu \mathrm{M})$ and the NO scavenger CPTIO $(300 \mu \mathrm{M})$ prevented development of SLES. The effect was reversed on washout of $\mathrm{L}-\mathrm{NMMA} / \mathrm{CP}$ TIO. B Spontaneous postsynaptic currents recorded in a CA3 pyramidal cell under control and low- $\mathrm{Mg}^{2+}$ conditions. The cell was clamped to $-50 \mathrm{mV}$, and inward and outward currents represent EPSCs and IPSCS, respectively. Removal of $\mathrm{Mg}^{2+}$ resulted in an increase in EPSC amplitude as well as in IPSC frequency and amplitude. After a delay of $\sim 10 \mathrm{~min}$, the first SLE occurred (arrow). C, Spontaneous synaptic activity recorded in an N0-deprived CA3 pyramidal cell under control and low-Mg ${ }^{2+}$ condition. NO deprivation prevented the initiation of SLES, although interictal activity was still present. This effect was reversible after removal of L-NMMA/CPTIO, and the first SLE was initiated within a few minutes (arrow). $\boldsymbol{D}$, Interictal activity as recorded in a CA3 pyramidal cell in an NO-deprived slice culture. Interictal activity consisted of small packages of inhibitory and excitatory synaptic currents, which occurred at a frequency of $0.1-0.5 \mathrm{~Hz}$. From the left to the right, interictal events are presented at shorter timescales. E, Spontaneous synaptic activity recorded in an 0DQ-treated CA3 pyramidal cell under control and low- $\mathrm{Mg}^{2+}$ condition. Inhibition of SGC with $10 \mu \mathrm{M}$ ODQ did not prevent development of SLEs. fp, Field potential; Im, membrane current.

low- $\mathrm{Mg}^{2+}$ condition (supplemental Fig. 1, available at www. jneurosci.org as supplemental material) (see Materials and Methods). Nitrite levels were significantly increased in slice cultures expressing epileptiform activity for $4 \mathrm{~h}$, unambiguously indicating NO formation $\left(8.11 \pm 2.2\right.$ vs $0.23 \pm 0.1 \mu \mathrm{M}$ for low- $\mathrm{Mg}^{2+}$ and normal ACSF, respectively; $n=18$ slice cultures for each condition; $p=0.03$ ).

\section{Effects of NO deprivation on} epileptiform activity in rat hippocampal slice cultures

Next, we determined whether enhanced NO formation during the latent phase was crucial for development of epileptiform activity. To this end, slice cultures were deprived of NO before (20 to $40 \mathrm{~min}$ ) as well as during low- $\mathrm{Mg}^{2+}$ condition by incubation with the broad-spectrum NOS inhibitor L-NMMA $(200 \mu \mathrm{M})$. In the majority of experiments, we coapplied PTIO or its more water-soluble derivative cPTIO (300 $\mu \mathrm{M})$ to scavenge $\mathrm{NO}$ that might be released from donors formed by the reaction of peroxynitrite with glucose (Moro et al., 1995) or from $S$-nitosoglutathione, which may represent $\sim 4 \%$ of mitochondrial glutathione (Steffen et al., 2001). Interictal activity developed in all $\mathrm{NO}$-deprived slice cultures under low- $\mathrm{Mg}^{2+}$ condition. However, SLEs were initiated only in 25\% of slice cultures $(n=20)$ (Fig. 4$)$. This was in sharp contrast to control condition (low- $\mathrm{Mg}^{2+}$ ACSF without NO deprivation), in which $100 \%$ of slice cultures developed SLEs $(n=70 ; p<0.001)$. The effect of NO deprivation on SLE suppression was reversible because washout of L-NMMA and CPTIO after up to $40 \mathrm{~min}$ under low- $\mathrm{Mg}^{2+}$ condition led to rapid initiation of SLEs within 2-5 $\mathrm{min}$ in $93 \%$ of slice cultures $(n=15)$ (Fig. $4 A, C)$.

We also investigated whether NO formation was critical for maintenance of epileptiform activity. In these experiments, NO deprivation was started after the first SLE $(n=15)$ by bath application of L-NMMA and cPTIO. Interestingly, L-NMMA alone $(200 \mu \mathrm{M})$ or in combination with cPTIO $(300 \mu \mathrm{M})$ suppressed SLEs only in $14 \%$ of slice cultures $(n=7$; $p=0.091$; data not shown). Application of cPTIO alone $(150-600 \mu \mathrm{M})$ for up to 40 min suppressed initiation of SLEs in 25\% of the slice cultures $(n=8 ; p=0.009)$. These data suggest that NO formation during the latent phase is crucial for initiation of SLEs, whereas fully developed epileptiform activity is less sensitive to subsequent NO deprivation.

Because sGC accounts for many of the neuromodulatory effects of NO (Esplugues, 2002; Guix et al., 2005), we tested whether the effect of NO deprivation on SLE initiation was mediated by sGC. We applied the sGC inhibitor ODQ $(10 \mu \mathrm{M})$ continuously via the perfusion $\sim 30 \mathrm{~min}$ before and during low- $\mathrm{Mg}^{2+}$ condition. Neither the development of SLEs nor of interictal activity was impaired in the presence of ODQ $(n=7)$. In a subsequent set of experiments, slice cultures 
$(n=11)$ were preincubated with ODQ (100 $\mu \mathrm{M}, 20 \mathrm{~min}$ ), and recordings were made in the presence of $50 \mu \mathrm{M}$ ODQ. Under these conditions, the initiation of SLEs in low- $\mathrm{Mg}^{2+} \mathrm{ACSF}$ was blocked in only $18 \%$ of the slice cultures $(p=0.017)$. In slice cultures expressing SLEs in the presence of ODQ, SLE onset latency was not significantly prolonged compared with control $(10 \mu \mathrm{M}$ ODQ, $15.2 \pm 3.6 \mathrm{~min}$; 50 $\mu \mathrm{M}$ ODQ, $12.8 \pm 2.6 \mathrm{~min}$; control, $11.57 \pm 1.2 \mathrm{~min} ; p>0.05)$. These data suggest that sGC-dependent mechanisms do not significantly contribute to SLE initiation.

\section{Effects of NO deprivation on EPSCs and} IPSCs in rat hippocampal slice cultures

To elucidate the mechanism by which NO deprivation suppresses the initiation of SLEs, we did whole-cell voltage-clamp recordings in visually identified pyramidal cells in the CA3 region of slice cultures. The neurons were clamped to $-50 \mathrm{mV}$. At this potential, EPSCs and IPSCs were reflected by inward and outward currents, respectively (see Materials and Methods), and allowed to investigate the effects of NO deprivation on excitatory and inhibitory synaptic transmission. We compared four parameters (i.e., amplitude and frequency of spontaneous EPSCs and IPSCs) in three groups of slice cultures (untreated, ODQ-treated, NO-deprived) under control and low- $\mathrm{Mg}^{2+}$ conditions (Fig. 4).

In normal ACSF, $\mathrm{NO}$ deprivation $(n=$ 9) and blockade of sGC by ODQ (10 and $50 \mu \mathrm{M} ; n=17$ ) had no effect on the peak amplitude of EPSCs and IPSCs compared with untreated slice cultures $(n=17)$ (Fig. $5 A)$. However, NO deprivation decreased both EPSC IPSC frequency $(p=0.04$ 0.001 , respectively) (Fig. $5 B$ ).

Under low- $\mathrm{Mg}^{2+}$ condition, the amplitude of EPSCs and IPSCs significantly increased in untreated slice cultures (Fig. $5 A, B)$. Strikingly, NO deprivation suppressed the increase in amplitudes of both EPSCs and IPSCs ( $p=0.54$ and 0.1 , respectively). Consequently, the EPSC amplitudes were significantly smaller in NOdeprived slice cultures compared with untreated slice cultures $(p=0.03)$.

Although IPSC frequency increased significantly under low$\mathrm{Mg}^{2+}$ condition in both untreated and NO-deprived slice cultures $(p<0.001)$ (Fig. 5B), NO deprivation led to a significant reduction of IPSC frequency compared with untreated slice cultures $(p=0.001)$. ODQ treatment caused no significant changes in IPSC or EPSC frequency under low- $\mathrm{Mg}^{2+}$ condition compared with untreated slice cultures, substantiating our finding that the effect of NO on SLE initiation was not mediated by sGC. The effects of NO deprivation were even more evident when comparing postsynaptic currents in individual neurons $(n=2)$
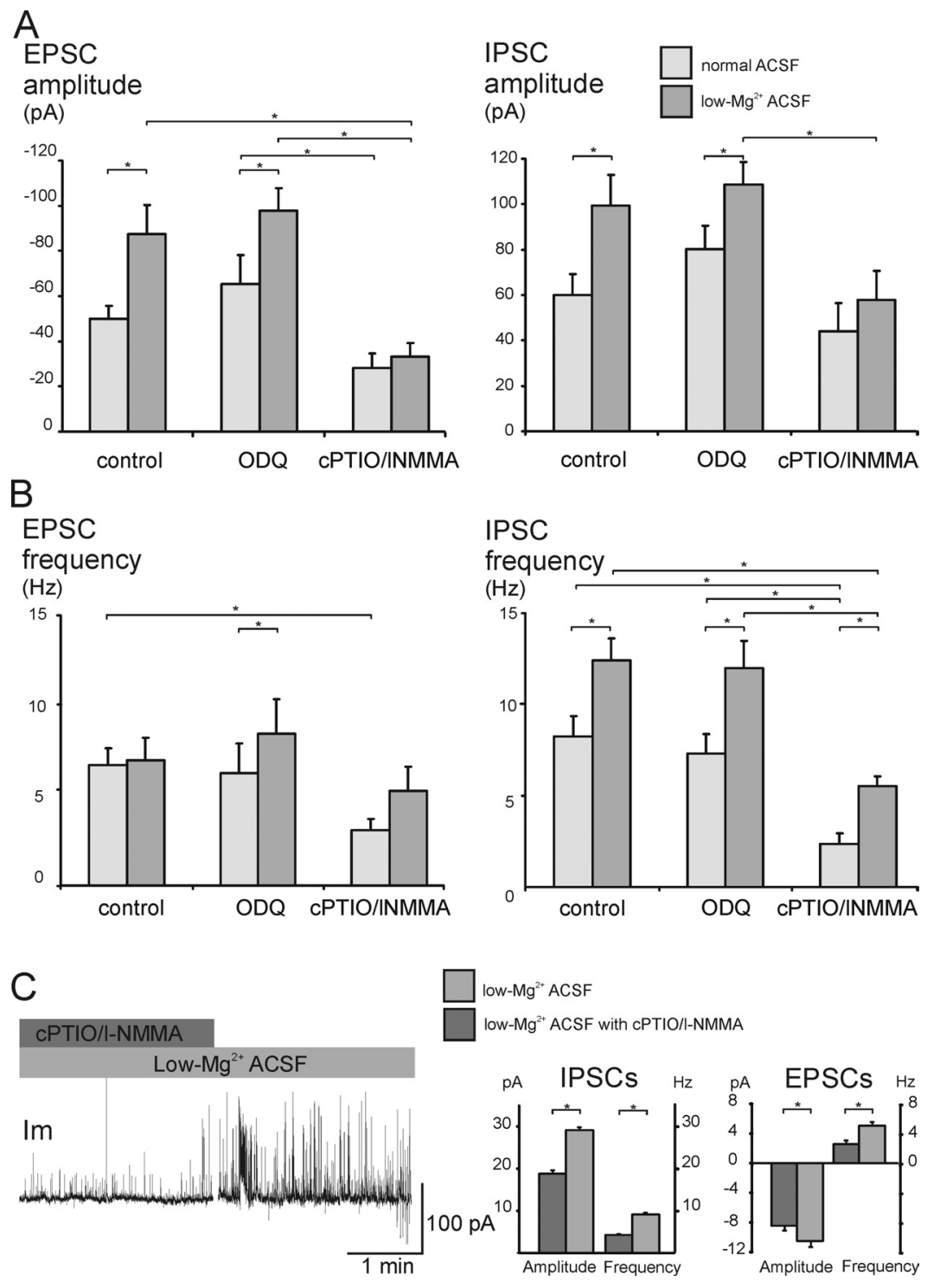

Figure 5. Effects of $\mathrm{sGC}$ inhibition and NO deprivation on spontaneous synaptic currents. $\boldsymbol{A}$, Effects of sGC inhibition ( $n=17$ cells) and NO deprivation ( $n=9$ cells) on the amplitude of EPSCs and IPSC s under control and low-Mg ${ }^{2+}$ conditions. Neither block of SGC nor NO deprivation had an effect on EPSC amplitude in normal ACSF compared with untreated slice cultures ( $n=17$ cells). EPSC and IPSC amplitude increased significantly in control and ODQ-treated under low- $\mathrm{Mg}^{2+}$ condition. In contrast, the increase in both EPSC and IPSC amplitudes was abolished in N0-deprived slice cultures. $\boldsymbol{B}$, Effects of SGC inhibition and N0 deprivation on the frequency of EPSCs and IPSCs under control and low-Mg ${ }^{2+}$ conditions. N0 deprivation significantly decreased EPSC frequency in normal ACSF as well as IPSC frequency under control and low- $\mathrm{Mg}^{2+}$ conditions. In contrast, IPSC and EPSC frequencies were not affected by $0 D Q$ treatment ( 10 and $50 \mu \mathrm{M}$ ). $C$, Effects of NO deprivation on spontaneous synaptic activity in the same cell under low-Mg ${ }^{2+}$ condition before and after washout of L-NMMA/CPTIO. Both the amplitude and the frequency of the EPSCS and IPSCS were significantly decreased in the presence of L-NMMA/CPTIO. Note that EPSC and IPSC amplitudes in the absence of L-NMMA/ CPTIO were on average still smaller than in untreated slice cultures under low- $\mathrm{Mg}^{2+}$ condition. IM, Membrane current.

under low- $\mathrm{Mg}^{2+}$ condition in the presence and absence of $\mathrm{L}^{-}$ NMMA/cPTIO. Amplitudes and frequencies of both EPSCs and IPSCs increased significantly after washout of L-NMMA/cPTIO (Fig. 5C).

These data suggest that NO formation in the latent phase under low- $\mathrm{Mg}^{2+}$ condition contributes to the enhancement of both excitatory and inhibitory synaptic transmission.

Effects of NO deprivation on epileptiform activity in hippocampal-entorhinal cortex slices from mice In a previous study, enhanced NO formation was described during epileptiform activity in acute hippocampal-entorhinal cor- 
A

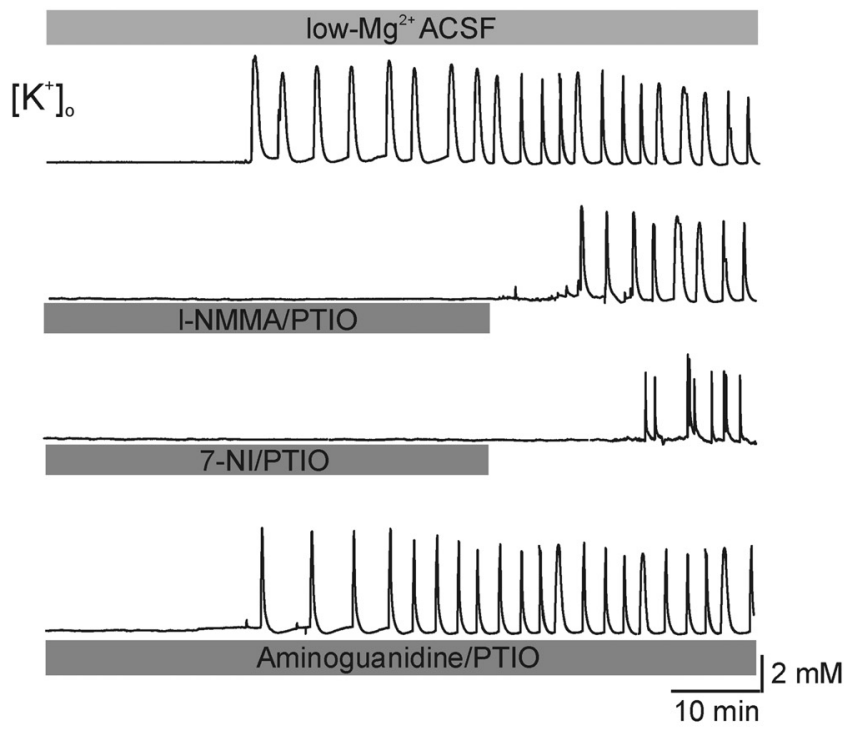

B

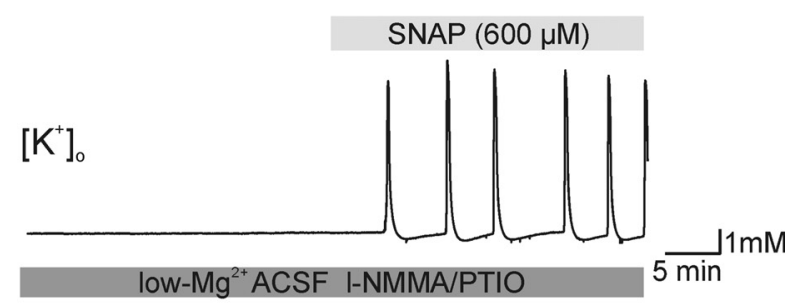

C

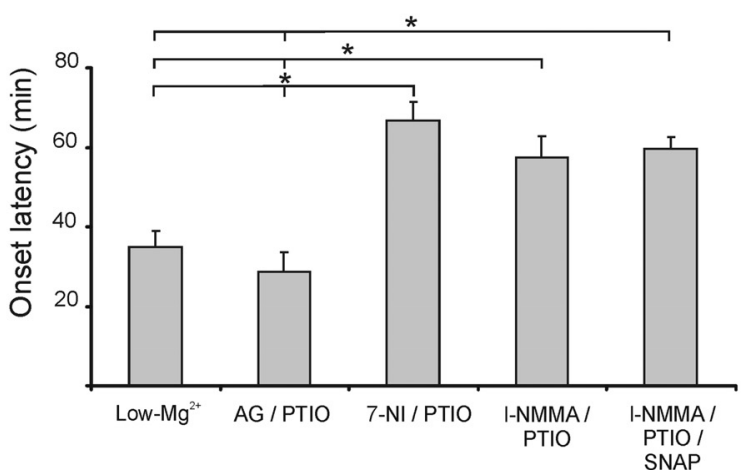

Figure 6. Effects of NO deprivation on the development of SLEs in hippocampal-entorhinal cortex slices from mice. $\boldsymbol{A}$, Comparison of the effects of different NOS inhibitors on the development of SLEs. Under low-Mg ${ }^{2+}$ condition, the onset latency of SLEs was $35 \pm 4 \mathrm{~min}(n=24)$ in untreated hippocampal-entorhinal cortex slices, as indicated by the appearance of transient increase in $\left[\mathrm{K}^{+}\right]_{0}$ (top trace). NO deprivation by L-NMMA/PTIO prevented development of SLEs. Similarly to L-NMMA, the nNOS inhibitor 7-NI (middle traces) but not the iNOS inhibitor aminoguanidine (AG) (bottom trace) prevented the development of SLEs in combination with PTIO. The effects were reversible after washout of the NOS inhibitors and PTIO. B, Application of L-NMMA/PTIO prevented the development of SLEs specifically by NO deprivation, because application of the NO donor SNAP initiated SLEs despite the presence of L-NMMA/PTIO. C, Diagram summarizing the SLE onset latencies in control and NO-deprived hippocampal-entorhinal cortex slices. The last column represents the group, in which SNAP was applied in the presence of L-NMMA/PTIO after $1 \mathrm{~h}$ of NO deprivation under low- $\mathrm{Mg}^{2+}$ condition.

tex slices from Wistar rats, and $\mathrm{NO}$ was shown to be required for maintenance of SLEs (Schuchmann et al., 2002). Because expression of nNOS in the entorhinal cortex is higher in rats than in mice (Blackshaw et al., 2003), we investigated whether the NO formation was still relevant for development and maintenance of epileptiform activity in the mouse entorhinal cortex. Under low$\mathrm{Mg}^{2+}$ condition, SLEs developed in $89 \%(n=27)$ (Fig. 6A, C) of the acute hippocampal-entorhinal cortex slices from wild-type mice, as revealed by field potential recordings and transients, rises in $\left[\mathrm{K}^{+}\right]_{\mathrm{o}}$. In the remaining slices, interictal activity was observed. The onset latency of SLE initiation was $35 \pm 4 \min (n=$ 24). Similar to our data from rat slice cultures, NO deprivation with L-NMMA $(200 \mu \mathrm{M})$ and PTIO $(300 \mu \mathrm{M})$ suppressed the initiation of SLEs in $100 \%$ of the acute slices $(n=11)$ for application times up to $1 \mathrm{~h}$ under low- $\mathrm{Mg}^{2+}$ condition (Fig. $6 A, C$ ), indicating that NO formation was crucial for SLE development also in mice. Washout of L-NMMA and PTIO led to rapid initiation of SLEs in $100 \%$ of the slices $(n=4)$. Moreover, SLEs were induced by bath application of the external NO-donor SNAP $(600 \mu \mathrm{M})$ in the presence of L-NMMA and PTIO in 100\% of the slices $(n=7)$ (Fig. $6 B$ ). The fact that application of exogenous NO was able to initiate SLEs clearly showed that the inhibitory effect of L-NMMA and PTIO was caused by NO deprivation rather than by nonspecific antiepileptic effect of the drugs.

To characterize the contribution of different types of NOS to the enhanced NO formation under low- $\mathrm{Mg}^{2+}$ condition, we investigated the effects of the inducible NOS (iNOS) inhibitor aminoguanidine $(200 \mu \mathrm{M})$ and the nNOS inhibitor 7-NI (100-200 $\mu \mathrm{M})$ in combination with PTIO. Aminoguanidine had no effect because SLEs developed in $100 \%$ of the slices $(n=9)$. Moreover, the SLE onset latency was not different compared with control $(n=22 ; p=0.4)$ (Fig. $6 A, C)$. These data also suggest that NO scavenging by PTIO alone is not sufficient to suppress SLE initi- ation. In contrast, 7-NI suppressed the initiation of SLEs in 100\% of the slices $(n=10)$ for up to $2 \mathrm{~h}$, which provided strong evidence that nNOS was the main source of NO under low- $\mathrm{Mg}^{2+}$ condition. Again, the application of SNAP in the presence of 7-NI and PTIO resulted in SLE initiation ( $n=2$; data not shown), indicating the specificity of the drugs in our model (Paul and Ekambaram, 2003; Luszczki et al., 2006).

There is evidence that bath application of the broad-spectrum NOS inhibitor L-NAME $(200 \mu \mathrm{M})$ interferes with the maintenance of SLEs in rat hippocampal-entorhinal cortex slices (Schuchmann et al., 2002). However, L-NAME (200-600 $\mu \mathrm{M}$ ) as applied after initiation of SLEs under low- $\mathrm{Mg}^{2+}$ condition had no effect on the maintenance of SLEs in $100 \%$ of the hippocampal-entorhinal cortex slices from mice $(n=6)$. This might indicate interspecies differences on the effects of NO on fully established epileptiform activity.

\section{Epileptiform activity in hippocampal-entorhinal cortex slices} from $n N^{-/-}$mice

To substantiate our pharmacological evidence for the role of nNOS in SLE initiation, we performed experiments in acute hippocampal-entorhinal cortex slices from $n \mathrm{NOS}^{-/-}$mice. Epileptiform activity in the entorhinal cortex (SLEs and interictal activity) developed in $64 \%$ of the acute slices $(n=25)$ from $n N O S^{-/-}$ mice (Fig. 7A), which was significantly less than in the slices from wild-type mice $(89 \% ; n=27 ; p=0.033)$. Strikingly, in those slices from $n N^{-1-}$ mice, in which we observed SLEs, the onset latency was significantly prolonged $(54.4 \pm 8$ vs $35 \pm 4$ min for $n N S^{-1-}$ ) and wild-type, respectively; $\left.p=0.027\right)$. In the remaining nine slices, stimulus trains $(20 \mathrm{~Hz}, 10 \mathrm{~s})$ as applied after $1 \mathrm{~h}$ in low- $\mathrm{Mg}^{2+}$ ACSF were associated with transient increases in $\left[\mathrm{K}^{+}\right]_{\mathrm{o}}$, indicating viability of the slices. However, electrical stimulation did not evoke SLEs. This observation was in sharp con- 
trast to slices from wild-type mice, in which stimulus trains under low- $\mathrm{Mg}^{2+}$ condition immediately evoked longlasting clonic afterdischarges (data not shown). Another striking difference was observed in the characteristics of SLEs in slices from $n \mathrm{NOS}^{-/-}$mice (Fig. $7 A, B$ ). Full-blown SLEs often alternated with SLEs lacking the DC shift. Moreover, periods of recurrent SLEs transformed to clonic discharges and vice versa (Fig. $7 A$, arrows). Using the standard deviation of SLE intervals as a measure of irregularity, we found significant differences between $n \mathrm{NOS}^{-/-}$and wild-type mice $(4.4 \pm 1$ vs $1.53 \pm 0.4 \min$ from $n=16$ and 24 , respectively; $p=0.022$ ).

$\mathrm{NO}$ is not completely absent in $n \mathrm{NOS}^{-/-}$mice because upregulation of eNOS (O’Dell et al., 1994) and alternative splice variants of nNOS (Eliasson et al., 1997) might partially counterbalance the effect of nNOS deletion. Thus, in a subsequent set of experiments, we applied the same NO-deprivation protocol (7-NI and PTIO) to slices from $n \mathrm{NOS}^{-1-}$ mice. SLEs developed in $37.5 \%$ of the slices from $n N^{-1-}$ mice $(n=8)$ in the presence of 7 -NI and PTIO, which was in sharp contrast to the complete block observed in slices from wild-type animals $(n=10 ; p=0.034)$. Moreover, the majority of the slices not expressing SLEs in the presence of 7-NI and PTIO did not initiate SLEs after removal of the drugs. In general, the occurrence of SLEs in slices from $n N^{-1-}$ mice was not significantly different in the presence or absence of 7-NI and PTIO $(p=0.18)$. These data strongly suggest that SLE initiation in $\mathrm{nNOS}^{-/-}$ mice becomes independent from $\mathrm{NO}$ formation. Additionally, a nonspecific antiepileptic effect of 7-NI and PTIO can be excluded.

Thus, nNOS deficiency in mice is associated with a decrease in seizure susceptibility, indicating that nNOS-mediated NO formation is crucial for both SLE initiation and regularity of recurrent SLEs in the mouse entorhinal cortex.

\section{Discussion}

Our main finding is that an elevated level of endogenous NO is crucial for the initiation of SLEs in brain slice preparations. NO formation contributes to the enhancement of synaptic activity under low $-\mathrm{Mg}^{2+}$ condition. This might provide a positive feedback for seizure initiation because the NO level increased already during the latent phase of epileptiform activity. This positive feedback represents a general mechanism because it was evident in different rodent species, preparations, and limbic structures.

B

C

$n \operatorname{NOS}(-/-)$
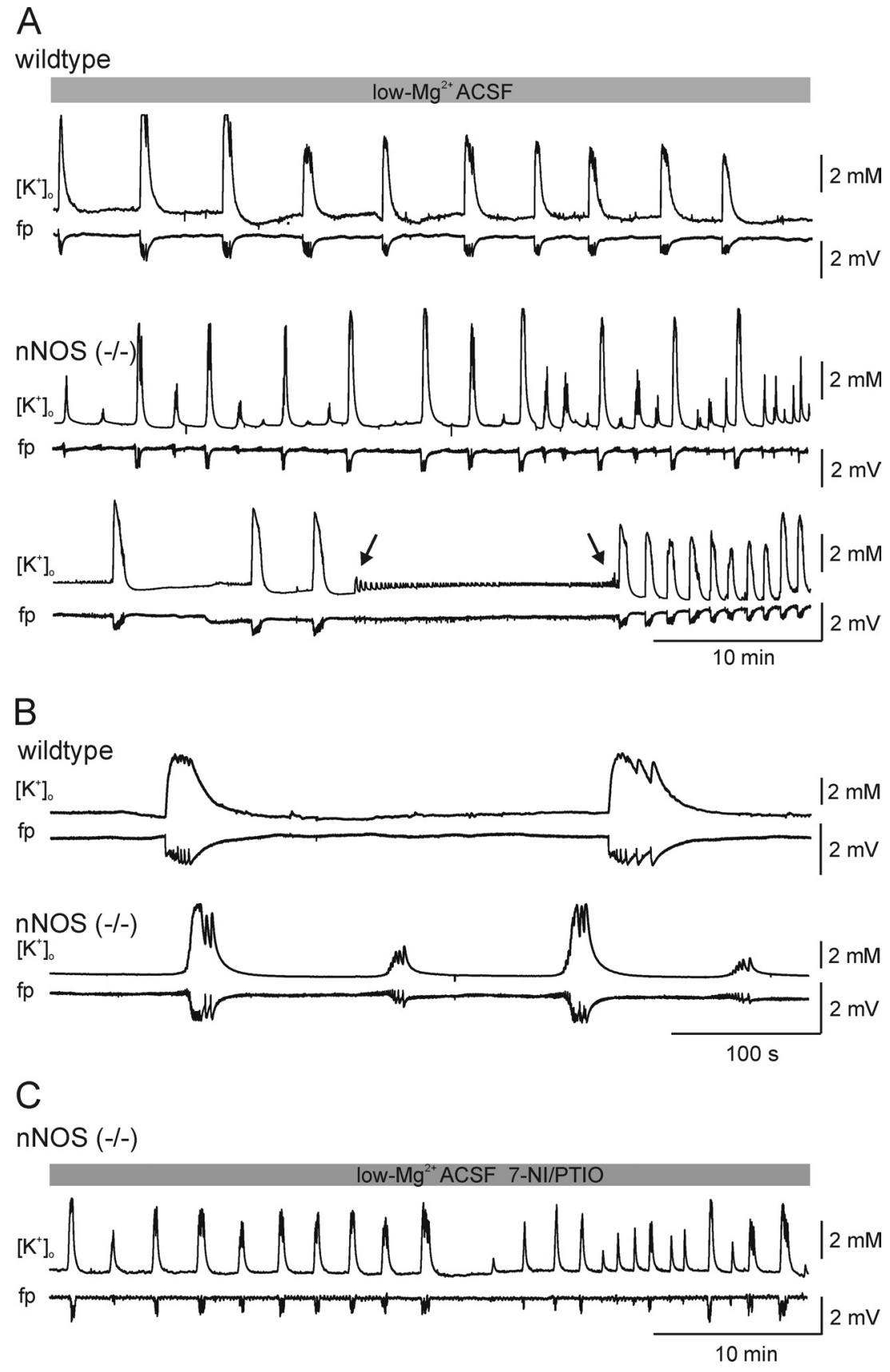

Figure 7. Characterization of SLEs in hippocampal entorhinal cortex slices from wild-type and nNOS ${ }^{-/-}$mice. A, Representative traces depicting SLE-associated changes in field potential and $\left[\mathrm{K}^{+}\right]_{0}$ in slices from wild-type (top 2 traces) and $n N \mathrm{NO}^{-/-}$ (bottom 4 traces) mice. SLEs of similar lengths and amplitudes recurred regularly in slices from wild-type mice. In contrast, SLE interval and the amplitude of the accompanied changes in $\left[\mathrm{K}^{+}\right]_{0}$ were more variable in slices from $n \mathrm{NOS}^{-/-}$mice. The SD of the SLE interval was significantly higher in slices from $n N^{-1-}$ mice compared with wild type. Arrows mark transition from recurrent SLEs to clonic discharges and vice versa. B, Characteristics of typical SLEs in slices from wild-type (top traces) and nNOS ${ }^{-1-}$ (bottom traces) mice at a shorter timescale. Note the presence of intermittent SLEs lacking of the DC shift between regular SLEs in the example trace from $n \mathrm{NOS}^{-1-}$ mice. Representative traces depicting SLE-associated changes in field potential and $\left[\mathrm{K}^{+}\right]_{0}$ in a slice from $n N^{-1-}$ mice in the presence of 7-NI and PTIO. Note that SLE characteristics in NO-deprived slices were similar to the SLEs in slices without 7-NI and PTIO, indicating that SLE initiation is independent on NO in $n N^{-1} S^{-1-}$ mice. fp, Field potential. 
seizure initiation (Dzhala and Staley, 2003). Although the number of neurons was relatively low, the massive nNOS-positive fiber network suggested that these neurons modulate synaptic activity over a large area (Ding et al., 2004). Indeed, the NO adduct of both DAF-fm and DAQ were rather homogeneously distributed within a given hippocampal layer. Moreover, the rate of $\mathrm{NO}$ formation was higher in stratum radiatum than in stratum pyramidale, which might reflect that the fiber network is the primary source of NO. At present, we cannot exclude that eNOS also contributes to NO formation in slice cultures. However, we observed DAF-fm accumulation in vascular structures containing eNOS (Keynes et al., 2004) only after more than $2 \mathrm{~h}$ of incubation with the fluorescent probe (R. Kovács and O. Kann, unpublished observation). The presence of DAF-fm in microglial cells might rather represent phagocytosis of dye particles than $\mathrm{NO}$ formation by iNOS (Fig. 2C, arrowheads), whose expression is upregulated only under exceptional conditions in rat slice cultures (Duport and Garthwaite, 2005). Comparing the effects of nNOS and iNOS inhibitors in mouse entorhinal cortex slices further strengthened the evidence that nNOS is the primary source of enhanced NO formation during epileptiform activity. Because the subtype specificity of NOS inhibitors is often a matter of debate (Alderton et al., 2001), we also examined SLE initiation in acute slices from $n N^{-1-}$ mice. Here we demonstrated an increased threshold for SLE initiation and irregular patterns of epileptiform activity. Therefore, we conclude that the increase in NO formation during epileptiform activity in limbic structures is mediated by nNOS. This conclusion is likely not restricted to the low $-\mathrm{Mg}^{2+}$ model of epilepsy because increased NO formation has been also demonstrated in epilepsy models in vivo (Kaneko et al., 2002; Gupta and Dettbarn, 2003; Kato et al., 2005). Thus, enhancement of NO formation might provide a general mechanism for seizure initiation.

\section{Targets of endogenous NO in slice preparations}

We provide evidence that there is a tonic formation of endogenous NO in slice cultures, which increases synaptic transmission because pharmacological NO deprivation decreased the IPSC and EPSC frequency in normal ACSF. Strikingly, under low$\mathrm{Mg}^{2+}$ condition, the enhanced $\mathrm{NO}$ formation contributes to the increase of both excitatory and inhibitory synaptic transmission. This is in line with previous reports demonstrating that NO interferes with both glutamatergic (Prast et al., 1998) and GABAergic (Wall, 2003) neurotransmission and influences neurotransmitter release depending on the type of synapse (Meffert et al., 1996; Mironov and Langohr, 2007; Wang et al., 2007). NO increases the release of glutamate by modulating presynaptic $\mathrm{N}$-type $\mathrm{Ca}^{2+}$ channels (Huang et al., 2003) and the release of GABA by an elevation of the intracellular $\mathrm{Ca}^{2+}$ concentration via cyclic adenosine diphosphate ribose/ryanodine-sensitive stores (Wang et al., 2006). The increase in transmitter release might also be related to NO-mediated acceleration of recycling of synaptic vesicles (Micheva et al., 2003). The aforementioned mechanisms involve the sGC/cGMP signal transduction pathway. In our study, however, the sGC inhibitor ODQ $(10 \mu \mathrm{M})$ did not mimic the effects of NO deprivation. The slight effect of ODQ at higher concentration $(50 \mu \mathrm{M})$ might represent unspecific interactions with other hem-proteins (Feelisch et al., 1999). Thus, the effect of NO on SLE initiation has to be mediated by alternative mechanisms. Likely candidates are ADP ribosylation or S-nitrosylation of proteins as well as other oxidative actions of NO or its derivatives that result from the reaction with superoxide (Schuman et al., 1994; Kleppisch et al., 1999; Trabace and Kendrick, 2000; Jaffrey et al., 2001). Such actions might be particularly important under epileptic conditions when superoxide formation is also enhanced (Kovács et al., 2002). This also explains the inability of cPTIO to prevent SLE initiation, because the reaction of $\mathrm{NO}$ with cPTIO results in formation of $\mathrm{NO}_{2}$ radical, which is still able to nitrosylate proteins (Goldstein et al., 2003).

There are several mechanisms by which $S$-nitrosylation might increase synaptic activity: (1) activation of the L-type $\mathrm{Ca}^{2+}$ channels (Tjong et al., 2007), (2) enhanced surface expression of AMPA receptors (Huang et al., 2005), (3) activation of persistent sodium current in hippocampal neurons (Hammarström and Gage, 1999), or (4) increased glutamate release via action on presynaptic proteins (Meffert et al., 1996). Moreover, $S$-nitrosylation might cause significant inhibition of mitochondrial complex I activity, which has been proposed to play a crucial role in epilepsy (Kunz et al., 2000).

\section{Effects of endogenous NO on SLE initiation}

Based on in vivo data, $\mathrm{NO}$ was postulated to be either proepileptic or antiepileptic (Wojtal et al., 2003). Broad-spectrum NOS inhibitors aggravated kainic acid-induced seizures, which was likely mediated by eNOS (Kato et al., 2005), and, in contrast, seizures were attenuated by nNOS inhibition (Yasuda et al., 2001). Increased nNOS activity was observed during the prodromal period of picrotoxin-induced seizures in the rat hippocampus. Pretreatment with 7-NI delayed the onset of the status epilepticus and was associated with a decrease in the activity of nNOS (Rajasekaran et al., 2003). NOS inhibitors increased whereas an NO donor decreased the antiepileptic effect of adenosine in the pentylenetetrazol model of epilepsy (Akula et al., 2008). Interestingly, L-NAME and 7-NI were equally effective, whereas aminoguanidine was not. However, the interpretation of in vivo data is difficult because of the complex effects of NO on cerebral blood flow and thus tissue oxygenation. In our model, in which these effects were absent, endogenous NO was clearly proepileptic regardless of animal species and type of preparation. Interestingly, NO exerted its effect already during the latent phase under low- $\mathrm{Mg}^{2+}$ condition and was less crucial for the maintenance of SLEs. We propose that enhanced NO formation induces long-lasting changes in synaptic transmission that cannot be revoked once SLEs have been initiated. Interestingly, the induction of certain forms of synaptic plasticity in the hippocampus is NO mediated by recruiting sGC-dependent (Bon and Garthwaite, 2003; Hopper and Garthwaite, 2006) or sGC-independent (Schuman et al., 1994; Kleppisch et al., 1999; Stanton et al., 2005) mechanisms. Thus, it is likely that long-term enhancement of synaptic transmission during the latent phase of epileptiform activity also facilitates SLE initiation. It is noteworthy that interictal activity was still present in NO-deprived slice cultures, suggesting that $\mathrm{NO}$ influences the entrainment of dynamically coupled oscillators rather than the pacemaker activity itself. This is in line with the concept that freely diffusible NO is a good candidate for increasing synchrony of adjacent ensembles of pyramidal neurons (Dzhala and Staley, 2003; Ledo et al., 2005; Derchansky et al., 2006). SLE initiation might involve selective activation of certain interneuron populations, as suggested in human temporal lobe epilepsy (Wendling et al., 2005). Accordingly, the NO-mediated increase in IPSC frequency under low- $\mathrm{Mg}^{2+}$ condition might significantly contribute to SLE initiation.

The occurrence of SLEs in $64 \%$ of the slices from $n N^{-1-}$ mice might indicate alternative sources of NO formation. The nNOS gene product exists in two catalytically active splice variants, $\mathrm{nNOS} \alpha$ and $\mathrm{nNOS} \beta$, contributing to 90 and $10 \%$ of $\mathrm{NO}$ formation, respectively. In $n N O S^{-/-}$mice, $n N O S \alpha$ activity is 
absent, whereas the expression of nNOS $\beta$ is upregulated (Eliasson et al., 1997). Alternatively, eNOS might partially compensate for certain effects of nNOS deletion (O'Dell et al., 1994). However, in a subset of slices, SLEs still occurred despite pharmacological NO deprivation, indicating that SLE initiation becomes independent on $\mathrm{NO}$ in slices from $n \mathrm{NOS}^{-/-}$mice. However, prolonged onset latency and the high variability of SLE intervals implies that synchrony is decreased and the number of SLE generator sites is increased in the absence of nNOS activity (Tsau et al., 1999).

\section{Long-term effects of NO in epilepsy}

We propose that NO-dependent enhancement of synaptic transmission is a key promoting factor for the initiation of seizures. Additionally, NO might exert long-term effects in epilepsy. NOdependent inhibition of mitochondrial electron transport chain activity (Brown, 2001), disruption of the mitochondrial networks (Yuan et al., 2007), and blockade of mitochondrial trafficking (Rintoul et al., 2006) might contribute to the metabolic impairment as described for the epileptic hippocampus (Kunz et al., 2000; Kann et al., 2005). In the presence of superoxide, NO gives rise to highly toxic peroxynitrite, thus contributing to free radical-mediated damage after long-lasting epileptic activity (Kovács et al., 2002; Patel, 2004).

\section{References}

Akula KK, Dhir A, Kulkarni SK (2008) Nitric oxide signaling pathway in the anti-convulsant effect of adenosine against pentylenetetrazol-induced seizure threshold in mice. Eur J Pharmacol 587:129-134.

Alderton WK, Cooper CE, Knowles RG (2001) Nitric oxide synthases: structure, function and inhibition. Biochem J 357:593-615.

Archer S (1993) Measurement of nitric oxide in biological models. FASEB J 7:349-360

Balcerczyk A, Soszynski M, Bartosz G (2005) On the specificity of 4-amino5 -methylamino-2' $7^{\prime}$-difluorofluorescein as a probe for nitric oxide. Free Radic Biol Med 39:327-335.

Blackshaw S, Eliasson MJ, Sawa A, Watkins CC, Krug D, Gupta A, Arai T, Ferrante RJ, Snyder SH (2003) Species, strain and developmental variations in hippocampal neuronal and endothelial nitric oxide synthase clarify discrepancies in nitric oxide-dependent synaptic plasticity. Neuroscience 119:979-990.

Bon CL, Garthwaite J (2003) On the role of nitric oxide in hippocampal long-term potentiation. J Neurosci 23:1941-1948.

Bredt DS, Snyder SH (1989) Nitric oxide mediates glutamate-linked enhancement of cGMP levels in the cerebellum. Proc Natl Acad Sci U S A 86:9030-9033.

Brown GC (2001) Regulation of mitochondrial respiration by nitric oxide inhibition of cytochrome c oxidase. Biochim Biophys Acta 1504:46-57.

Brown LA, Key BJ, Lovick TA (1999) Bio-imaging of nitric oxide-producing neurones in slices of rat brain using 4,5-diaminofluorescein. J Neurosci Methods 92:101-110.

Burette A, Zabel U, Weinberg RJ, Schmidt HH, Valtschanoff JG (2002) Synaptic localization of nitric oxide synthase and soluble guanylyl cyclase in the hippocampus. J Neurosci 22:8961-8970.

Calabrese V, Bates TE, Stella AM (2000) NO synthase and NO-dependent signal pathways in brain aging and neurodegenerative disorders: the role of oxidant/antioxidant balance. Neurochem Res 25:1315-1341.

Derchansky M, Rokni D, Rick JT, Wennberg R, Bardakjian BL, Zhang L, Yarom Y, Carlen PL (2006) Bidirectional multisite seizure propagation in the intact isolated hippocampus: the multifocality of the seizure "focus". Neurobiol Dis 23:312-328.

de Vasconcelos AP, Bouilleret V, Riban V, Wasterlain C, Nehlig A (2005) Role of nitric oxide in cerebral blood flow changes during kainate seizures in mice: genetic and pharmacological approaches. Neurobiol Dis $18: 270-281$

Ding JD, Burette A, Nedvetsky PI, Schmidt HH, Weinberg RJ (2004) Distribution of soluble guanylyl cyclase in the rat brain. J Comp Neurol 472:437-448.

Duport S, Garthwaite J (2005) Pathological consequences of inducible nitric oxide synthase expression in hippocampal slice cultures. Neuroscience 135:1155-1166.

Dzhala VI, Staley KJ (2003) Transition from interictal to ictal activity in limbic networks in vitro. J Neurosci 23:7873-7880.

Eliasson MJ, Blackshaw S, Schell MJ, Snyder SH (1997) Neuronal nitric oxide synthase alternatively spliced forms: prominent functional localizations in the brain. Proc Natl Acad Sci U S A 94:3396-3401.

Espey MG, Miranda KM, Thomas DD, Wink DA (2001) Distinction between nitrosating mechanisms within human cells and aqueous solution. J Biol Chem 276:30085-30091.

Esplugues JV (2002) NO as a signalling molecule in the nervous system. Br J Pharmacol 135:1079-1095.

Feelisch M, Kotsonis P, Siebe J, Clement B, Schmidt HH (1999) The soluble guanylyl cyclase inhibitor $1 \mathrm{H}-[1,2,4]$ oxadiazolo[ $[4,3,-\mathrm{a}]$ quinoxalin-1one is a nonselective heme protein inhibitor of nitric oxide synthase and other cytochrome P-450 enzymes involved in nitric oxide donor bioactivation. Mol Pharmacol 56:243-253.

Garthwaite J, Charles SL, Chess-Williams R (1988) Endothelium-derived relaxing factor release on activation of NMDA receptors suggests role as intercellular messenger in the brain. Nature 336:385-388.

Goldstein S, Russo A, Samuni A (2003) Reactions of PTIO and carboxy$\mathrm{PTIO}$ with ${ }^{*} \mathrm{NO},{ }^{*} \mathrm{NO} 2$, and $\mathrm{O} 2{ }^{-*}$. J Biol Chem 278:50949-50955.

Good PF, Werner P, Hsu A, Olanow CW, Perl DP (1996) Evidence of neuronal oxidative damage in Alzheimer's disease. Am J Pathol 149:21-28.

Guix FX, Uribesalgo I, Coma M, Muñoz FJ (2005) The physiology and pathophysiology of nitric oxide in the brain. Prog Neurobiol 76:126-152.

Gundersen HJ (1986) Stereology of arbitrary particles. A review of unbiased number and size estimators and the presentation of some new ones, in memory of William R. Thompson. J Microsc 143:3-45.

Gupta RC, Dettbarn WD (2003) Prevention of kainic acid seizures-induced changes in levels of nitric oxide and high-energy phosphates by 7-nitroindazole in rat brain regions. Brain Res 981:184-192.

Hammarström AK, Gage PW (1999) Nitric oxide increases persistent sodium current in rat hippocampal neurons. J Physiol 520:451-461.

Heinemann U, Arens J (1992) Production and calibration of ion-sensitive microelectrodes. In: Practical electrophysiological methods (Kettenmann H, Grantyn R, eds), pp 206-212. New York: Wiley.

Hopper RA, Garthwaite J (2006) Tonic and phasic nitric oxide signals in hippocampal long-term potentiation. J Neurosci 26:11513-11521.

Huang CC, Chan SH, Hsu KS (2003) cGMP/protein kinase G-dependent potentiation of glutamatergic transmission induced by nitric oxide in immature rat rostral ventrolateral medulla neurons in vitro. Mol Pharmacol 64:521-532.

Huang PL, Dawson TM, Bredt DS, Snyder SH, Fishman MC (1993) Targeted disruption of the neuronal nitric oxide synthase gene. Cell 75:1273-1286.

Huang S, Heikal AA, Webb WW (2002) Two-photon fluorescence spectroscopy and microscopy of $\mathrm{NAD}(\mathrm{P}) \mathrm{H}$ and flavoprotein. Biophys J 82:2811-2825.

Huang Y, Man HY, Sekine-Aizawa Y, Han Y, Juluri K, Luo H, Cheah J, Lowenstein C, Huganir RL, Snyder SH (2005) S-nitrosylation of $\mathrm{N}$-ethylmaleimide sensitive factor mediates surface expression of AMPA receptors. Neuron 46:533-540.

Iadecola C, Li J, Ebner TJ, Xu X (1995) Nitric oxide contributes to functional hyperemia in cerebellar cortex. Am J Physiol 268:R1153-R1162.

Jaffrey SR, Erdjument-Bromage H, Ferris CD, Tempst P, Snyder SH (2001) Protein S-nitrosylation: a physiological signal for neuronal nitric oxide. Nat Cell Biol 3:193-197.

Jourd'heuil D (2002) Increased nitric oxide-dependent nitrosylation of 4,5diaminofluorescein by oxidants: implications for the measurement of intracellular nitric oxide. Free Radic Biol Med 33:676-684.

Kaneko K, Itoh K, Berliner LJ, Miyasaka K, Fujii H (2002) Consequences of nitric oxide generation in epileptic-seizure rodent models as studied by in vivo EPR. Magn Reson Med 48:1051-1056.

Kann O, Kovács R, Njunting M, Behrens CJ, Otáhal J, Lehmann TN, Gabriel S, Heinemann U (2005) Metabolic dysfunction during neuronal activation in the ex vivo hippocampus from chronic epileptic rats and humans. Brain 128:2396-2407.

Kato N, Sato S, Yokoyama H, Kayama T, Yoshimura T (2005) Sequential changes of nitric oxide levels in the temporal lobes of kainic acid-treated mice following application of nitric oxide synthase inhibitors and phenobarbital. Epilepsy Res 65:81-91. 
Keynes RG, Duport S, Garthwaite J (2004) Hippocampal neurons in organotypic slice culture are highly resistant to damage by endogenous and exogenous nitric oxide. Eur J Neurosci 19:1163-1173.

Kirkby RD, Carroll DM, Grossman AB, Subramaniam S (1996) Factors determining proconvulsant and anticonvulsant effects of inhibitors of nitric oxide synthase in rodents. Epilepsy Res 24:91-100.

Kleppisch T, Pfeifer A, Klatt P, Ruth P, Montkowski A, Fässler R, Hofmann F (1999) Long-term potentiation in the hippocampal CA1 region of mice lacking cGMP-dependent kinases is normal and susceptible to inhibition of nitric oxide synthase. J Neurosci 19:48-55.

Kovács R, Schuchmann S, Gabriel S, Kann O, Kardos J, Heinemann U (2002) Free radical-mediated cell damage after experimental status epilepticus in hippocampal slice cultures. J Neurophysiol 88:2909-2918.

Kunz WS, Kudin AP, Vielhaber S, Blümcke I, Zuschratter W, Schramm J, Beck H, Elger CE (2000) Mitochondrial complex I deficiency in the epileptic focus of patients with temporal lobe epilepsy. Ann Neurol 48:766-773.

Ledo A, Barbosa RM, Gerhardt GA, Cadenas E, Laranjinha J (2005) Concentration dynamics of nitric oxide in rat hippocampal subregions evoked by stimulation of the NMDA glutamate receptor. Proc Natl Acad Sci U S A 102:17483-17488.

Luhmann HJ, Dzhala VI, Ben-Ari Y (2000) Generation and propagation of 4-AP-induced epileptiform activity in neonatal intact limbic structures in vitro. Eur J Neurosci 12:2757-2768.

Luszczki JJ, Sacharuk A, Wojciechowska A, Andres-Mach MM, DudraJastrzebska M, Mohamed M, Sawicka KM, Kozinska J, Czuczwar SJ (2006) 7-Nitroindazole enhances dose-dependently the anticonvulsant activities of conventional antiepileptic drugs in the mouse maximal electroshock-induced seizure model. Pharmacol Rep 58:660-671.

Meffert MK, Calakos NC, Scheller RH, Schulman H (1996) Nitric oxide modulates synaptic vesicle docking fusion reactions. Neuron 16:1229-1236.

Micheva KD, Buchanan J, Holz RW, Smith SJ (2003) Retrograde regulation of synaptic vesicle endocytosis and recycling. Nat Neurosci 6:925-932.

Mironov SL, Langohr K (2007) Modulation of synaptic and channel activities in the respiratory network of the mice by NO/cGMP signalling pathways. Brain Res 1130:73-82.

Mody I, Lambert JD, Heinemann U (1987) Low extracellular magnesium induces epileptiform activity and spreading depression in rat hippocampal slices. J Neurophysiol 57:869-888.

Moro MA, Darley-Usmar VM, Lizasoain I, Su Y, Knowles RG, Radomski MW, Moncada S (1995) The formation of nitric oxide donors from peroxynitrite. Br J Pharmacol 116:1999-2004.

Mülsch A, Busse R, Mordvintcev PI, Vanin AF, Nielsen EO, Scheel-Krüger J, Olesen SP (1994) Nitric oxide promotes seizure activity in kainatetreated rats. Neuroreport 5:2325-2328.

Nagano T (1999) Practical methods for detection of nitric oxide. Luminescence 14:283-290.

Namiki S, Kakizawa S, Hirose K, Iino M (2005) NO signalling decodes frequency of neuronal activity and generates synapse-specific plasticity in mouse cerebellum. J Physiol 566:849-863.

O'Dell TJ, Huang PL, Dawson TM, Dinerman JL, Snyder SH, Kandel ER, Fishman MC (1994) Endothelial NOS and the blockade of LTP by NOS inhibitors in mice lacking neuronal NOS. Science 265:542-546.

Patel M (2004) Mitochondrial dysfunction and oxidative stress: cause and consequence of epileptic seizures. Free Radic Biol Med 37:1951-1962.

Patzak A, Steege A, Lai EY, Brinkmann JO, Kupsch E, Spielmann N, Gericke A, Skalweit A, Stegbauer J, Persson PB, Seeliger E (2008) Angiotensin II response in afferent arterioles of mice lacking either the endothelial or neuronal isoform of nitric oxide synthase. Am J Physiol Regul Integr Comp Physiol 294:R429-R437.

Paul V, Ekambaram P (2003) Effect of 7-nitroindazole alone and in combination with phenobarbitone and diazepam on picrotoxin-induced convulsions in rats. Indian J Physiol Pharmacol 47:400-406.

Prast H, Philippu A (2001) Nitric oxide as modulator of neuronal function. Prog Neurobiol 64:51-68.

Prast H, Tran MH, Fischer H, Philippu A (1998) Nitric oxide-induced release of acetylcholine in the nucleus accumbens: role of cyclic GMP, glutamate, and GABA. J Neurochem 71:266-273.

Rajasekaran K, Jayakumar R, Venkatachalam K (2003) Increased neuronal nitric oxide synthase (nNOS) activity triggers picrotoxin-induced seizures in rats and evidence for participation of nNOS mechanism in the action of antiepileptic drugs. Brain Res 979:85-97.

Rintoul GL, Bennett VJ, Papaconstandinou NA, Reynolds IJ (2006) Nitric oxide inhibits mitochondrial movement in forebrain neurons associated with disruption of mitochondrial membrane potential. J Neurochem 97:800-806.

Sato M, Nakajima T, Goto M, Umezawa Y (2006) Cell-based indicator to visualize picomolar dynamics of nitric oxide release from living cells. Anal Chem 78:8175-8182.

Schuchmann S, Albrecht D, Heinemann U, von Bohlen und Halbach O (2002) Nitric oxide modulates low- $\mathrm{Mg}^{2+}$-induced epileptiform activity in rat hippocampal-entorhinal cortex slices. Neurobiol Dis 11:96-105.

Schuman EM, Meffert MK, Schulman H, Madison DV (1994) An ADPribosyltransferase as a potential target for nitric oxide action in hippocampal long-term potentiation. Proc Natl Acad Sci USA 91:11958-11962.

Sheng JZ, Wang D, Braun AP (2005) DAF-FM (4-amino-5-methylamino$2^{\prime}, 7^{\prime}$-difluorofluorescein) diacetate detects impairment of agoniststimulated nitric oxide synthesis by elevated glucose in human vascular endothelial cells: reversal by vitamin C and L-sepiapterin. J Pharmacol Exp Ther 315:931-940.

Shu SY, Ju G, Fan LZ (1988) The glucose oxidase-DAB-nickel method in peroxidase histochemistry of the nervous system. Neurosci Lett 85:169-171.

Stanton PK, Winterer J, Zhang XL, Müller W (2005) Imaging LTP of presynaptic release of FM1-43 from the rapidly recycling vesicle pool of Schaffer collateral-CA1 synapses in rat hippocampal slices. Eur J Neurosci 22:2451-2461.

Steffen M, Sarkela TM, Gybina AA, Steele TW, Trasseth NJ, Kuehl D, Giulivi C (2001) Metabolism of S-nitrosoglutathione in intact mitochondria. Biochem J 356:395-402.

Szabadits E, Cserép C, Ludányi A, Katona I, Gracia-Llanes J, Freund TF, Nyíri G (2007) Hippocampal GABAergic synapses possess the molecular machinery for retrograde nitric oxide signalling. J Neurosci 27:8101-8111.

Tjong YW, Jian K, Li M, Chen M, Gao TM, Fung ML (2007) Elevated endogenous nitric oxide increases $\mathrm{Ca}^{2+}$ flux via L-type $\mathrm{Ca}^{2+}$ channels by $\mathrm{S}$-nitrosylation in rat hippocampal neurons during severe hypoxia and in vitro ischemia. Free Radic Biol Med 42:52-63.

Trabace L, Kendrick KM (2000) Nitric oxide can differentially modulate striatal neurotransmitter concentrations via soluble guanylate cyclase and peroxynitrite formation. J Neurochem 75:1664-1674.

Tsau Y, Guan L, Wu JY (1999) Epileptiform activity can be initiated in various neocortical layers: an optical imaging study. J Neurophysiol 82:1965-1973.

Valtschanoff JG, Weinberg RJ, Kharazia VN, Nakane M, Schmidt HH (1993) Neurons in rat hippocampus that synthesize nitric oxide. J Comp Neurol 331:111-121.

Wall MJ (2003) Endogenous nitric oxide modulates GABAergic transmission to granule cells in adult rat cerebellum. Eur J Neurosci 18:869-878.

Wang S, Teschemacher AG, Paton JF, Kasparov S (2006) Mechanism of nitric oxide action on inhibitory GABAergic signaling within the nucleus tractus solitarii. FASEB J 20:1537-1539.

Wang S, Paton JF, Kasparov S (2007) Differential sensitivity of excitatory and inhibitory synaptic transmission to modulation by nitric oxide in rat nucleus tractus solitarii. Exp Physiol 92:371-382.

Wendling F, Hernandez A, Bellanger JJ, Chauvel P, Bartolomei F (2005) Interictal to ictal transition in human temporal lobe epilepsy: insights from a computational model of intracerebral EEG. J Clin Neurophysiol 22:343-356.

Wojtal K, Gniatkowska-Nowakowska A, Czuczwar SJ (2003) Is nitric oxide involved in the anticonvulsant action of antiepileptic drugs? Pol J Pharmacol 55:535-542.

Yasuda H, Fujii M, Fujisawa H, Ito H, Suzuki M (2001) Changes in nitric oxide synthesis and epileptic activity in the contralateral hippocampus of rats following intrahippocampal kainate injection. Epilepsia 42:13-20.

Yuan H, Gerencser AA, Liot G, Lipton SA, Ellisman M, Perkins GA, BossyWetzel E (2007) Mitochondrial fission is an upstream and required event for bax foci formation in response to nitric oxide in cortical neurons. Cell Death Differ 14:462-471. 